\title{
Low-frequency Carbon Radio Recombination Lines. I. Calculations of Departure Coefficients
}

\author{
F. Salgado ${ }^{1}$, L. K. Morabito ${ }^{1}$, J. B. R. Oonk ${ }^{1,2}$, P. Salas ${ }^{1}$, M. C. Toribio ${ }^{1}$, \\ H. J. A. Röttgering ${ }^{1}$, and A. G. G. M. Tielens ${ }^{1}$ \\ ${ }^{1}$ Leiden Observatory, University of Leiden, P.O. Box 9513, 2300 RA Leiden, The Netherlands \\ ${ }^{2}$ Netherlands Institute for Radio Astronomy (ASTRON), Postbus 2, 7990 AA Dwingeloo, The Netherlands \\ Received 2015 August 3; revised 2017 January 16; accepted 2017 January 30; published 2017 March 13
}

\begin{abstract}
In the first paper of this series, we study the level population problem of recombining carbon ions. We focus our study on high quantum numbers, anticipating observations of carbon radio recombination lines to be carried out by the Low Frequency Array. We solve the level population equation including angular momentum levels with updated collision rates up to high principal quantum numbers. We derive departure coefficients by solving the level population equation in the hydrogenic approximation and including low-temperature dielectronic capture effects. Our results in the hydrogenic approximation agree well with those of previous works. When comparing our results including dielectronic capture, we find differences that we ascribe to updates in the atomic physics (e.g., collision rates) and to the approximate solution method of the statistical equilibrium equations adopted in previous studies. A comparison with observations is discussed in an accompanying article, as radiative transfer effects need to be considered.
\end{abstract}

Key words: ISM: atoms - ISM: general - line: formation - methods: numerical - radio lines: general - radio lines: ISM

\section{Introduction}

The interplay of stars and their surrounding gas leads to the presence of distinct phases in the interstellar medium (ISM) of galaxies (e.g., Field et al. 1969; McKee \& Ostriker 1977). Diffuse atomic clouds (the cold neutral medium, CNM) have densities of about $50 \mathrm{~cm}^{-3}$ and temperatures of about $80 \mathrm{~K}$, where atomic hydrogen is largely neutral but carbon is singly ionized by photons with energies between 11.2 and $13.6 \mathrm{eV}$. The warmer $(\sim 8000 \mathrm{~K})$ and more tenuous $\left(\sim 0.5 \mathrm{~cm}^{-3}\right)$ intercloud phase is heated and ionized by far ultraviolet (FUV) and extreme ultraviolet (EUV) photons escaping from $\mathrm{H}$ II regions (Wolfire et al. 2003), usually referred to as the warm neutral medium (WNM) and warm ionized medium (WIM). The phases of the ISM are often globally considered to be in thermal equilibrium and in pressure balance (Savage \& Sembach 1996; Cox 2005). However, the observed large turbulent width and presence of gas at thermally unstable, intermediate temperatures attests to the importance of heating by kinetic energy input. In addition, the ISM also hosts molecular clouds, where hydrogen is in the form of $\mathrm{H}_{2}$ and selfgravity plays an important role. All of these phases are directly tied to key questions on the origin and evolution of the ISM, including the energetics of the CNM, WNM, and the WIM; the evolutionary relationship of atomic and molecular gas; the relationship of these ISM phases with newly formed stars; and the conversion of their radiative and kinetic power into thermal and turbulent energy of the ISM (e.g., Elmegreen \& Scalo 2004; Scalo \& Elmegreen 2004; Cox 2005; McKee \& Ostriker 2007).

The neutral phases of the ISM have been studied using optical and UV observations of atomic lines. These observations can provide the physical conditions but are limited to pinpoint experiments toward bright background sources and are hampered by dust extinction (Snow \& McCall 2006). At radio wavelengths, dust extinction is not important, and observations of the $21 \mathrm{~cm}$ hyperfine transition of neutral atomic hydrogen have been used to study the neutral phases (e.g., Weaver \& Williams 1973; Heiles \& Troland 2003b; Kalberla et al. 2005). On a global scale, these observations have revealed the prevalence of the two-phase structure in the ISM of cold clouds embedded in a warm intercloud medium, but they have also pointed out challenges to this theoretical view (Kulkarni \& Heiles 1987, p. 87; Kalberla \& Kerp 2009). It has been notoriously challenging to determine the physical characteristics (density, temperature) of the neutral structures in the ISM because separating the cold and warm components is challenging (e.g., Heiles \& Troland 2003a). In this context, carbon radio recombination lines (CRRLs) provide a promising tracer of the neutral phases of the ISM (e.g., Peters et al. 2011; Oonk et al. 2015a).

Carbon has a lower ionization potential $(11.2 \mathrm{eV})$ than hydrogen $(13.6 \mathrm{eV})$ and can be ionized by radiation fields in regions where hydrogen is largely neutral. Recombination of carbon ions with electrons to high Rydberg states will lead to CRRLs in the submillimeter to decameter wavelength range. CRRLs have been observed in the ISM of our Galaxy toward two types of clouds: diffuse clouds (e.g., Konovalenko \& Sodin 1981; Erickson et al. 1995; Roshi et al. 2002; Stepkin et al. 2007; Oonk et al. 2014) and photodissociation regions (PDRs), the boundaries of $\mathrm{HII}$ regions and their parent molecular clouds (e.g., Natta et al. 1994; Wyrowski et al. 1997; Quireza et al. 2006). The first low-frequency (26.1 MHz) CRRL was detected in absorption toward the supernova remnant Cas A by Konovalenko \& Sodin (1980) (wrongly attributed to a hyperfine structure line of ${ }^{14} \mathrm{~N}$, Konovalenko \& Sodin 1981). This line corresponds to a transition occurring at high quantum levels $(n=631)$. Recently, Stepkin et al. (2007) detected CRRLs in the range 25.5-26.5 MHz toward Cas A, corresponding to transitions involving levels as large as $n=1009$. 
Observations of low-frequency carbon recombination lines can be used to probe the physical properties of the diffuse ISM. However, detailed modeling is required to interpret the observations. Watson et al. (1980), Walmsley \& Watson (1982) showed that, at low temperatures $\left(T_{e} \lesssim 100 \mathrm{~K}\right)$, electrons can recombine with carbon ions by simultaneously exciting the ${ }^{2} P_{1 / 2}-{ }^{2} P_{3 / 2}$ fine structure line, a process known as dielectronic capture. ${ }^{3}$ Such a process occurs to high $n$ states and can explain the behavior of the high- $n$ CRRLs observed toward Cas A. Walmsley \& Watson (1982) modified the code from Brocklehurst \& Salem (1977) to include dielectronic recombination. Payne et al. (1994) modified the code to consider transitions up to 10,000 levels. All of these results assume a statistical distribution of the angular momentum levels, an assumption that is not valid at intermediate levels for low temperatures. Moreover, the lower the temperature, the higher the $n$ level for which that assumption is not valid.

The increased sensitivity, spatial resolution, and bandwidth of the Low Frequency Array (LOFAR; van Haarlem et al. 2013) is opening the low-frequency sky to systematic studies of high quantum number radio recombination lines. The recent detection of high-level CRRLs using LOFAR toward the line of sight of Cas A (Asgekar et al. 2013), Cyg A (Oonk et al. 2014), and the first extragalactic detection in the starburst galaxy M82 (Morabito et al. 2014) illustrate the potential of LOFAR for such studies. Moreover, pilot studies have demonstrated that surveys of lowfrequency radio recombination lines of the galactic plane are within reach, providing a new and powerful probe of the diffuse ISM. These new observations have motivated us to reassess some of the approximations made by previous works and to expand the range of applicability of recombination line theory in terms of physical parameters. In addition, increased computer power allows us to solve the level population problem considering a much larger number of levels than ever before. Furthermore, updated collision rates are now available (Vrinceanu et al. 2012), allowing us to explicitly consider the level population of quantum angular momentum sublevels to high principal quantum number levels. Finally, it can be expected that the Square Kilometer Array (SKA) will further revolutionize our understanding of the low-frequency universe with even higher sensitivity and angular resolution (Oonk et al. 2015a).

In this work, we present the method to calculate the level population of recombining ions and provide some example results applicable to low-temperature diffuse clouds in the ISM. In an accompanying article (Salgado et al. 2017, from here on Paper II), we will present results specifically geared toward radio recombination line studies of the diffuse ISM. In Section 2, we introduce the problem of level population of atoms and the methods to solve this problem for hydrogen and hydrogenic carbon atoms. We also present the rates used in this work to solve the level population problem. In Section 3, we discuss our results, focusing on hydrogen and carbon atoms. We compare our results in terms of the departure coefficients with previous results from the literature. In Section 4, we summarize our results and provide the conclusions of the present work.

3 This process has been referred to in the astronomical literature as dielectronic-like recombination or just dielectronic recombination. Strictly speaking, dielectronic recombination refers to dielectronic capture followed by stabilization. Dielectronic capture refers to the capture of the electron in an excited $n$ state accompanied by simultaneous excitation of the ${ }^{2} P_{1 / 2}$ core electron to the excited ${ }^{2} P_{3 / 2}$ state. The captured electron can either autoionize, be collisionally transferred to another state, or radiatively decay.

\section{Theory}

A large fraction of our understanding of the physical processes in the universe comes from observations of atomic lines in astrophysical plasmas. In order to interpret the observations, accurate models for the level population of atoms are needed as the strength (or depth) of an emission (absorption) line depends on the level population of atoms. Here, we summarize the basic ingredients needed to build level population models and provide a basic description of the level population problem. We begin our discussion by describing the line emission and absorption coefficients $\left(j_{\nu}\right.$ and $k_{\nu}$, respectively), which are given by (Shaver 1975; Gordon \& Sorochenko 2009)

$$
\begin{gathered}
j_{\nu}=\frac{h \nu}{4 \pi} A_{n^{\prime} n} N_{n^{\prime}} \phi(\nu), \\
k_{\nu}=\frac{h \nu}{4 \pi}\left(N_{n} B_{n n^{\prime}}-N_{n^{\prime}} B_{n^{\prime} n}\right) \phi(\nu),
\end{gathered}
$$

where $h$ is the Planck constant, $N_{n^{\prime}}$ is the level population of a given upper level $\left(n^{\prime}\right)$, and $N_{n}$ is the level population of the lower level $(n) ; \phi(\nu)$ is the line profile, $\nu$ is the frequency of the transition, and $A_{n^{\prime} n}$ and $B_{n^{\prime} n}\left(B_{n n^{\prime}}\right)$ are the Einstein coefficients for spontaneous and stimulated emission (absorption), ${ }^{4}$ respectively.

Under local thermodynamic equilibrium (LTE) conditions, level populations are given by the Saha-Boltzmann equation (e.g., Brocklehurst 1971):

$$
\begin{aligned}
N_{n l}(\mathrm{LTE}) & =N_{e} N_{\text {ion }}\left(\frac{h^{2}}{2 \pi m_{e} k T_{e}}\right)^{3 / 2} \frac{\omega_{n l}}{2 \omega_{i}} e^{\chi_{n},} \\
\chi_{n} & =\frac{\mathrm{hc} R y Z^{2}}{n^{2} k T_{e}},
\end{aligned}
$$

where $T_{e}$ is the electron temperature, $N_{e}$ is the electron density in the nebula, $N_{\text {ion }}$ is the ion density, $m_{e}$ is the electron mass, $k$ is the Boltzmann constant, $h$ is the Planck constant, $c$ is the speed of light, and $R y$ is the Rydberg constant; $\omega_{n l}$ is the statistical weight of the level $n$ and angular quantum momentum level $l\left[\omega_{n l}=2(2 l+1)\right.$, for hydrogen], and $\omega_{i}$ is the statistical weight of the parent ion. The factor $\left(h^{2} / 2 \pi m_{e} k T_{e}\right)^{1 / 2}$ is the thermal de Broglie wavelength, $\Lambda\left(T_{e}\right)$, of the free electron. ${ }^{5}$ In the most general case, lines are formed under non-LTE conditions, and the level population equation must be solved in order to properly model the line properties as a function of quantum level $(n)$ :

Following, for example, Seaton (1959a) and Brocklehurst (1970), we present the results of our modeling in terms of the departure coefficients $\left(b_{n l}\right)$, defined by

$$
b_{n l}=\frac{N_{n l}}{N_{n l}(\mathrm{LTE})},
$$

and $b_{n}$ values are computed by taking the weighted sum of the $b_{n l}$ values:

$$
b_{n}=\sum_{l=0}^{n-1}\left(\frac{2 l+1}{n^{2}}\right) b_{n l}
$$

\footnotetext{
4 We provide the formulation to obtain the values for the rates in Appendix C. $5 \Lambda\left(T_{e}\right)^{3} \approx 4.14133 \times 10^{-16} T_{e}^{-1.5} \mathrm{~cm}^{3}$.
} 
Note that, at a given $n$, the $b_{n l}$ values for large $l$ levels influence the final $b_{n}$ value the most due to the statistical weight factor. At low frequencies, stimulated emission is important (Goldberg 1966), and we introduce the correction factor for stimulated emission as defined by Brocklehurst \& Seaton (1972):

$$
\beta_{n, n^{\prime}}=\frac{1-\left(b_{n^{\prime}} / b_{n}\right) \exp \left(-h \nu / k T_{e}\right)}{1-\exp \left(-h \nu / k T_{e}\right)} .
$$

Unless otherwise stated, the $\beta_{n}$ values presented here correspond to $\alpha$ transitions $\left(n^{\prime}=n+1 \rightarrow n\right)$. The description of the level population in terms of departure coefficients is convenient as it reduces the level population problem to a more easily handled problem, as we will show in Section 2.1 .

\subsection{Level Population of Carbon Atoms under Non-LTE Conditions}

The observations of high- $n$ carbon recombination lines in the ISM motivated Watson et al. (1980) to study the effect on the level population of dielectronic capture and its inverse process (autoionization) in low-temperature $\left(T_{e} \lesssim 100 \mathrm{~K}\right)$ gas. Watson et al. (1980) used $l$-changing collision rates $^{6}$ from Jacobs \& Davis (1978) and concluded that, for levels $n \approx 250-300$, dielectronic capture for carbon ions can be of importance. In a later work, Walmsley \& Watson (1982) used collision rates from Dickinson (1981) and estimated a value for which autoionization becomes more important than angular momentum changing rates. The change in collision rates led them to conclude that the influence of dielectronic capture on the $b_{n}$ values is important at levels $n \gtrsim 300$. Clearly, the results are sensitive to the choice of the angular momentum changing rates. Here, we will explicitly consider $l$ sublevels when solving the level population equation.

The dielectronic capture and autoionization processes affect only the $\mathrm{C}^{+}$ions in the ${ }^{2} P_{3 / 2}$ state, so we treat the level populations for the two ion cores in the ${ }^{2} P_{1 / 2}$ states separately in the evaluation of the level population (Walmsley \& Watson 1982). The equations for carbon atoms recombining to the ${ }^{2} P_{3 / 2}$ ion core population have to include terms describing dielectronic capture $\left(\alpha_{n l}^{d}\right)$ and autoionization $\left(A_{n l}^{a}\right)$ :

$$
\begin{aligned}
& b_{n l}\left[\sum_{n^{\prime}<n} \sum_{l^{\prime}=l \pm 1} A_{n l n^{\prime} l^{\prime}}+\sum_{n^{\prime} \neq n}\left(B_{n l n^{\prime} l^{\prime}} I_{\nu}+C_{n l n^{\prime} l^{\prime}}\right)\right. \\
& \left.+\sum_{l^{\prime}=l \pm 1} C_{n l n l^{\prime}}+A_{n l}^{a}+C_{n l, i}\right] \\
& =\sum_{n^{\prime}>n} \sum_{l^{\prime}=l \pm 1} b_{n^{\prime} l^{\prime}} \frac{\omega_{n^{\prime} l^{\prime}}}{\omega_{n l}} e^{\Delta \chi_{n^{\prime} n}} A_{n^{\prime} l^{\prime} n l} \\
& +\sum_{n^{\prime} \neq n} \sum_{l^{\prime}=l \pm 1} b_{n^{\prime} l^{\prime}} \frac{\omega_{n^{\prime} l^{\prime}}}{\omega_{n l}} e^{\Delta \chi_{n^{\prime} n}}\left(B_{n^{\prime} l^{\prime} n l} I_{\nu}+C_{n^{\prime} l^{\prime} n l}\right) \\
& +\sum_{l^{\prime}=l \pm 1} b_{n l^{\prime}}\left(\frac{\omega_{n l^{\prime}}}{\omega_{n l}}\right) C_{n l^{\prime} n l}+\frac{N_{e} N_{3 / 2}^{+}}{N_{n l}(\mathrm{LTE})}\left(\alpha_{n l}+C_{i, n l}\right) \\
& +\frac{N_{e} N_{1 / 2}^{+}}{N_{n l}(\mathrm{LTE})} \alpha_{n l}^{d} .
\end{aligned}
$$

\footnotetext{
6 We use the term $l$-changing collision rates to refer to collision rates that induce a transition from state $n l$ to $n l \pm 1$.
}

The left-hand side of Equation (7) describes all of the processes that take an electron out of the $n l$ level, and the right-hand side the processes that add an electron to the $n l$ level; $A_{n n^{\prime} l^{\prime}}$ is the coefficient for spontaneous emission, $B_{n n^{\prime} l^{\prime}}$ is the coefficient for stimulated emission or absorption induced by a radiation field $I_{\nu} ; C_{n l n^{\prime} l^{\prime}}$ is the coefficient for energy-changing collisions (i.e., transitions with $\left.n \neq n^{\prime}\right), C_{n l n l^{\prime}}$ is the coefficient for $l$-changing collisions, $C_{n l, i}\left(C_{i, n l}\right)$ is the coefficient for collisional ionization (three-body recombination); and $\alpha_{n l}$ is the coefficient for radiative recombination. A description of the coefficients entering into Equation (7) is given in Section 2.3 and in further detail in the appendix. The level population equation is solved by finding the values for the departure coefficients. The level population for carbon ions recombining to the ${ }^{2} P_{1 / 2}$ level is hydrogenic, and we solve for the departure coefficients $\left(b_{n l}^{1 / 2}\right)$ using Equation (7), ignoring the coefficients for dielectronic capture and autoionization.

After computing the $b_{n l}^{1 / 2}$ and $b_{n l}^{3 / 2}$, we compute the departure coefficients $\left(b_{n}^{1 / 2}\right.$ and $\left.b_{n}^{3 / 2}\right)$ for both parent ion populations by summing over all $l$ states (Equation (5)). The final departure coefficients for carbon are obtained by computing the weighted average of both ion cores:

$$
b_{n}^{\text {final }}=\frac{b_{n}^{1 / 2}+b_{n}^{3 / 2}\left[N_{3 / 2}^{+} / N_{1 / 2}^{+}\right]}{1+\left[N_{3 / 2}^{+} / N_{1 / 2}^{+}\right]} .
$$

Note that, in order to obtain the final departure coefficients, the relative population of the parent ion cores is needed. Here, we assume that the population ratio of the two ion cores $N_{3 / 2}^{+}$to $N_{1 / 2}^{+}$is determined by collisions with electrons and hydrogen atoms. This ratio can be obtained using (Ponomarev \& Sorochenko 1992; Payne et al. 1994)

$$
\begin{gathered}
R=\frac{N_{3 / 2}^{+} / N_{1 / 2}^{+}}{N_{3 / 2}^{+}(\mathrm{LTE}) / N_{1 / 2}^{+}(\mathrm{LTE})} \\
=\frac{N_{e} \gamma_{e}+N_{H} \gamma_{H}}{N_{e} \gamma_{e}+N_{H} \gamma_{H}+A_{3 / 2,1 / 2}},
\end{gathered}
$$

where $\gamma_{e}=4.51 \times 10^{-6} T_{e}^{-1 / 2} \mathrm{~cm}^{-3} \mathrm{~s}^{-1}$ is the de-excitation rate due to collisions with electrons, $\gamma_{H}=5.8 \times 10^{-10}$ $T_{e}^{0.02} \mathrm{~cm}^{-3} \mathrm{~s}^{-1}$ is the de-excitation rate due to collisions with hydrogen atoms (Payne et al. 1994), ${ }^{7} N_{\mathrm{H}}$ is the atomic hydrogen density, and $A_{3 / 2,1 / 2}=2.4 \times 10^{-6} \mathrm{~s}^{-1}$ is the spontaneous radiative decay rate of the core. In this work, we have ignored collisions with molecular hydrogen, which should be included for high-density PDRs. Collision rates for $\mathrm{H}_{2}$ excitation of $\mathrm{C}^{+}$have been calculated by Flower (1988). In the cases of interest here, the value of $R$ is dominated by collisions with atomic hydrogen. We recognize that the definition of $R$ given in Equation (9) is related to the critical density $\left(N_{\mathrm{cr}}\right)$ of a two-level system by $R=1 /\left(1+N_{\mathrm{cr}} / N_{X}\right)$, where $N_{X}$ is the density of the collision partner (electron or hydrogen). The LTE ratio of the ion core is given by the

\footnotetext{
Payne et al. (1994) used rates from Tielens \& Hollenbach (1985), based on Launay \& Roueff (1977) for collisions with hydrogen atoms and Hayes \& Nussbaumer (1984) for collisions with electrons. Newer rates are available for collisions with electrons (Wilson \& Bell 2002) and hydrogen atoms (Barinovs et al. 2005), but the difference in values is negligible.
} 

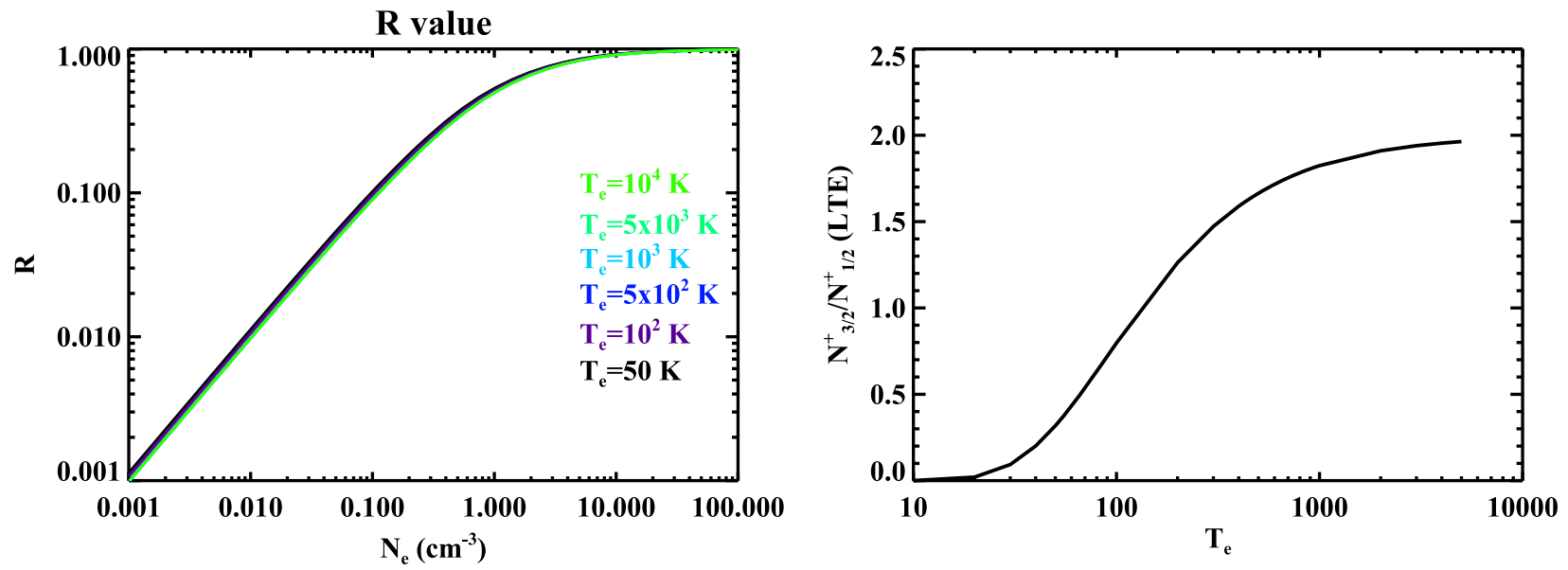

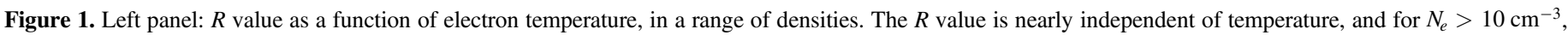
$R \approx 1$. Right panel: ion LTE ratios as a function of $T_{e}$, independent of density.

statistical weights of the levels and the temperature $\left(T_{e}\right)$ of the gas:

$$
\frac{N_{3 / 2}^{+}(\mathrm{LTE})}{N_{1 / 2}^{+}(\mathrm{LTE})}=\frac{g_{3 / 2}}{g_{1 / 2}} e^{-\Delta E / k T_{e}},
$$

where $g_{3 / 2}=4, g_{1 / 2}=2$ are the statistical weights of the fine structure levels, and $\Delta E=92 \mathrm{~K}$ is the energy difference of the fine structure transition. The LTE level population ratio as a function of temperature is shown in Figure 1, illustrating the strong dependence on temperature of this value. At densities below the critical density $\left(\approx 300 \mathrm{~cm}^{-3}\right.$ for collisions with $\left.\mathrm{H}\right)$, the fine structure levels fall out of LTE, and the value for $R$ becomes very small (Figure 1). Note that $R$ is not very sensitive to the temperature.

With the definition of $R$ given above, the final departure coefficient can be written as (Ponomarev \& Sorochenko 1992)

$$
b_{n}^{\text {final }}=\frac{b_{n}^{1 / 2}+b_{n}^{3 / 2} R\left[N_{3 / 2}^{+} / N_{1 / 2}^{+}\right]_{\mathrm{LTE}}}{1+R\left[N_{3 / 2}^{+} / N_{1 / 2}^{+}\right]_{\mathrm{LTE}}} .
$$

The final departure coefficient is the value that we are interested in to describe CRRLs.

\subsection{Numerical Method}

Having described how to derive the $b_{n}^{\text {final }}$, now we focus on the problem of obtaining the departure coefficients for both ion cores from the level population equation. We use the same procedure to obtain the departure coefficients for both parent ion cores, as the only difference in the level population equation for the ${ }^{2} P_{3 / 2}$ and the ${ }^{2} P_{1 / 2}$ cores is the inclusion of dielectronic recombination and autoionization processes. We will refer to $b_{n l}$ and $b_{n}$ without making a distinction between ${ }^{2} P_{3 / 2}$ and ${ }^{2} P_{1 / 2}$ in this subsection.

We follow the methods described in Brocklehurst (1971) and improved in Hummer \& Storey (1987) to solve the level population equation in an iterative manner. First, we solve the level population equation by assuming that the $l$ sublevels are in statistical equilibrium, that is, $b_{n}=b_{n l}$ for all $l$ sublevels. We refer to this approach as the $n$ method (see Appendix B). Second, we use the previously computed values to determine the coefficients on the right-hand side of Equation (7) that contain terms with $n^{\prime} \neq n$. Thus, the level population equation for a given $n$ is a tridiagonal equation on the $l$ sublevels involving terms of the type $l \pm 1$. This tridiagonal equation is solved for the $b_{n l}$ values (further details are given in Appendix B). The second step of this procedure is repeated until the difference between the computed departure coefficients is less than $1 \%$.

We consider a fixed maximum number of levels, $n_{\max }$, equal to 9900 . We make no explicit assumptions on the asymptotic behavior of the $b_{n}$ for larger values of $n$. Therefore, no fitting or extrapolation is required for large $n$. The adopted value for $n_{\max }$ is large enough for the asymptotic limit $-b_{n} \rightarrow 1$ for $n>n_{\max }$ - to hold even at the lowest densities considered here. For the $n l$ method, we need to consider all $l$ sublevels up to a high level ( $n \sim 1000)$. For levels higher than this critical $n$ level $\left(n_{\text {crit }}\right)$, we assume that the $l$ sublevels are in statistical equilibrium. In our calculations, $n_{\text {crit }}=1500$, regardless of the density.

\subsection{Rates Used in This Work}

In this section, we provide a brief description of the rates used in solving the level population. Further details and the mathematical formulations for each rate are given in Appendices C, D, E, and F. Accurate values for the rates are critical to obtain meaningful departure coefficients when solving the level population equation (Equation (7)). Radiative rates are known to high accuracy $(<1 \%)$ as they can be computed from first principles. On the other hand, collision rates at low temperatures are more uncertain $(\sim 20 \%$, Vriens \& Smeets 1980).

\subsubsection{Einstein A and B Coefficients}

The Einstein coefficients for spontaneous and stimulated transitions can be derived from first principles. We used the recursion formula described in Storey \& Hummer (1991) to obtain the values for the Einstein $A_{n n^{\prime} l^{\prime}}$ coefficients. To solve the $n$ method (our first step in solving the level population equation), we require the values for $A_{n n^{\prime}}$, which can be easily obtained by summing the $A_{n l n^{\prime} l^{\prime}}$ :

$$
A_{n n^{\prime}}=\frac{1}{n^{2}} \sum_{l^{\prime}=0}^{n-1} \sum_{l=l^{\prime} \pm 1}(2 l+1) A_{n l n^{\prime} l^{\prime}} .
$$


The mathematical formulation to obtain values for spontaneous transitions is detailed in Appendix C.

The coefficients for stimulated emission and absorption $\left(B_{n n^{\prime}}\right)$ are related to the $A_{n n^{\prime}}$ coefficients by

$$
\begin{aligned}
& B_{n n^{\prime}}=\frac{c^{2}}{2 h \nu^{3}} A_{n n^{\prime}}, \\
& B_{n^{\prime} n}=\left(\frac{n}{n^{\prime}}\right)^{2} B_{n n^{\prime}} .
\end{aligned}
$$

\subsubsection{Energy-changing Collision Rates}

In general, energy-changing collisions are dominated by the interactions of electrons with the atom. The interaction of an electron with an atom can induce transitions of the type

$$
X_{n l}+e^{-} \rightleftarrows X_{n^{\prime} l^{\prime}}+e^{-},
$$

with $n^{\prime} \neq n$ changing the distribution of electrons in an atom population. Hummer \& Storey (1987) used the formulation of Percival \& Richards (1978). The collision rates derived by Percival \& Richards (1978) are essentially the same as that from Gee et al. (1976). However, the collision rates from Gee et al. (1976) are not valid for the low temperatures of interest here. Instead, we use collision rates from Vriens \& Smeets (1980). We note that at high $T_{e}$ and for high $n$ levels, the Bethe (Born) approximation holds, and values of the rates from Vriens \& Smeets (1980) differ by less than 20\% when compared to those from Gee et al. (1976). The good agreement between the two rates is expected since the results from Vriens \& Smeets (1980) are based on Gee et al. (1976). On the other hand, at low $T_{e}$ and for low $n$-level values, the two rates differ by several orders of magnitude, and, indeed, the Gee et al. (1976) values are too high to be physically realistic. A comparison of the rates for different values of $T_{e}$ and $n \rightarrow n+\Delta n$ transitions is shown in Figure 2. We explore the effects of using Vriens \& Smeets (1980) rates on the $b_{n}$ values in Section 3.2.

The inverse rates are obtained from the detailed balance:

$$
C_{n^{\prime} n}=\left(\frac{n}{n^{\prime}}\right)^{2} e^{\chi_{n}-\chi_{n^{\prime}}} C_{n n^{\prime}}
$$

In order to solve the $n l$ method, rates of the type $C_{n l n^{\prime} l^{\prime}}$ with $n \neq n^{\prime}$ are needed. Here, the approach of Hummer \& Storey (1987) is followed, and the collision rates are normalized by the oscillator strength of the transitions (Equation (5) in Hummer $\&$ Storey 1987). Only transitions with $\Delta l=1$ were included as these dominate the collision process (Hummer \& Storey 1987).

\subsubsection{Angular Momentum Changing Collision Rates}

For low $n$ levels, the $l$-level population has to be explicitly calculated. Moreover, for the dielectronic capture process, the angular momentum changing collisions set the value for which the dielectronic capture process is important, and transitions of the type

$$
X_{n l}+\mathrm{C}^{+} \rightleftarrows X_{n l \pm 1}+\mathrm{C}^{+}
$$

must be considered. In general, collisions with ions are more important than collisions with electrons. Here, for simplicity, we adopt that $\mathrm{C}^{+}$is the dominant cation.
Hummer \& Storey (1987) used $l$-changing collision rates from Pengelly \& Seaton (1964), which are computed iteratively for a given $n$ level starting at $l=0$ or $l=n-1$. However, as pointed out by Hummer \& Storey (1987) and Brocklehurst (1971), the values for the $l$-changing rates obtained by starting the iterations at $l=0$ differ from those obtained when starting at $l=n-1$. Moreover, averaging the $l$-changing rates obtained by the two different initial conditions leads to an oscillatory behavior of the rates that depends on $l$ (Brocklehurst 1970). Hummer \& Storey (1987) circumvented this problem by normalizing the value of the rates by the oscillator strength (Equation (4) in Hummer \& Storey 1987). In addition, at high $n$ levels and high densities, the values for $C_{n l n^{\prime} l^{\prime}}$ can become negative (Equation (43) in Pengelly \& Seaton 1964). This poses a problem when studying the level population of carbon atoms at the high $n$ levels of interest in the present work. ${ }^{8}$ The more recent study of Vrinceanu et al. (2012) provides a general formulation to obtain the value of $l$-changing transition rates. These new rates use a much smaller cutoff radius of the probability of the transition for large impact parameters. Furthermore, the rates from Vrinceanu et al. (2012) are well behaved over a large range of temperatures and densities, and they do not exhibit the oscillatory behavior with $l$ sublevel shown by the Pengelly \& Seaton (1964) rates. Therefore, we use the Vrinceanu et al. (2012) rates in this work. Vrinceanu et al. (2012) derived the following expression, valid for $n>10$ and $n \sqrt{T_{e}}<2.4 \times 10^{4} \mathrm{~K}^{1 / 2}$ :

$$
\begin{aligned}
C_{n l \rightarrow n l+1}= & 12 \sqrt{\pi} a_{0}^{3}(2 \pi c R y) \sqrt{\left(\frac{\mathrm{hcRy}}{k T_{e}}\right)\left(\frac{\mu}{m_{e}}\right)} n^{4} \\
& \times\left[1-\left(\frac{l}{n}\right)^{2}\left(\frac{2 l+3}{2 l+1}\right)\right] .
\end{aligned}
$$

where $a_{0}$ is the Bohr radius and $\mu$ is the reduced mass of the system. Values for the inverse process are obtained by using the detailed balance:

$$
C_{n l+1 \rightarrow n l}=\frac{(2 l+1)}{(2 l+3)} C_{n l \rightarrow n l+1} .
$$

We note that the $l$-changing collision rates obtained by using the formula from Vrinceanu et al. (2012) can differ by a factor of 6 (Vrinceanu et al. 2012) from those using the Pengelly \& Seaton (1964) formulation. We discuss the effect on the final $b_{n}$ values in Section 3.2, where we compare our results with those of Storey \& Hummer (1995) in the hydrogenic approximation and with those of Ponomarev \& Sorochenko (1992) for carbon atoms.

\subsubsection{Radiative Recombination}

Radiative ionization occurs when an excited atom absorbs a photon with enough energy to ionize the excited electron. The process can be represented as follows:

$$
X_{n l}+h \nu \rightleftarrows X^{+}+e^{-},
$$

and the inverse process is radiative recombination. We use the recursion relation described in Storey \& Hummer (1991) to

\footnotetext{
8 We note that this was not a problem for Hummer \& Storey (1987), since they assumed a statistical distribution of the $l$ levels for high $n$.
} 

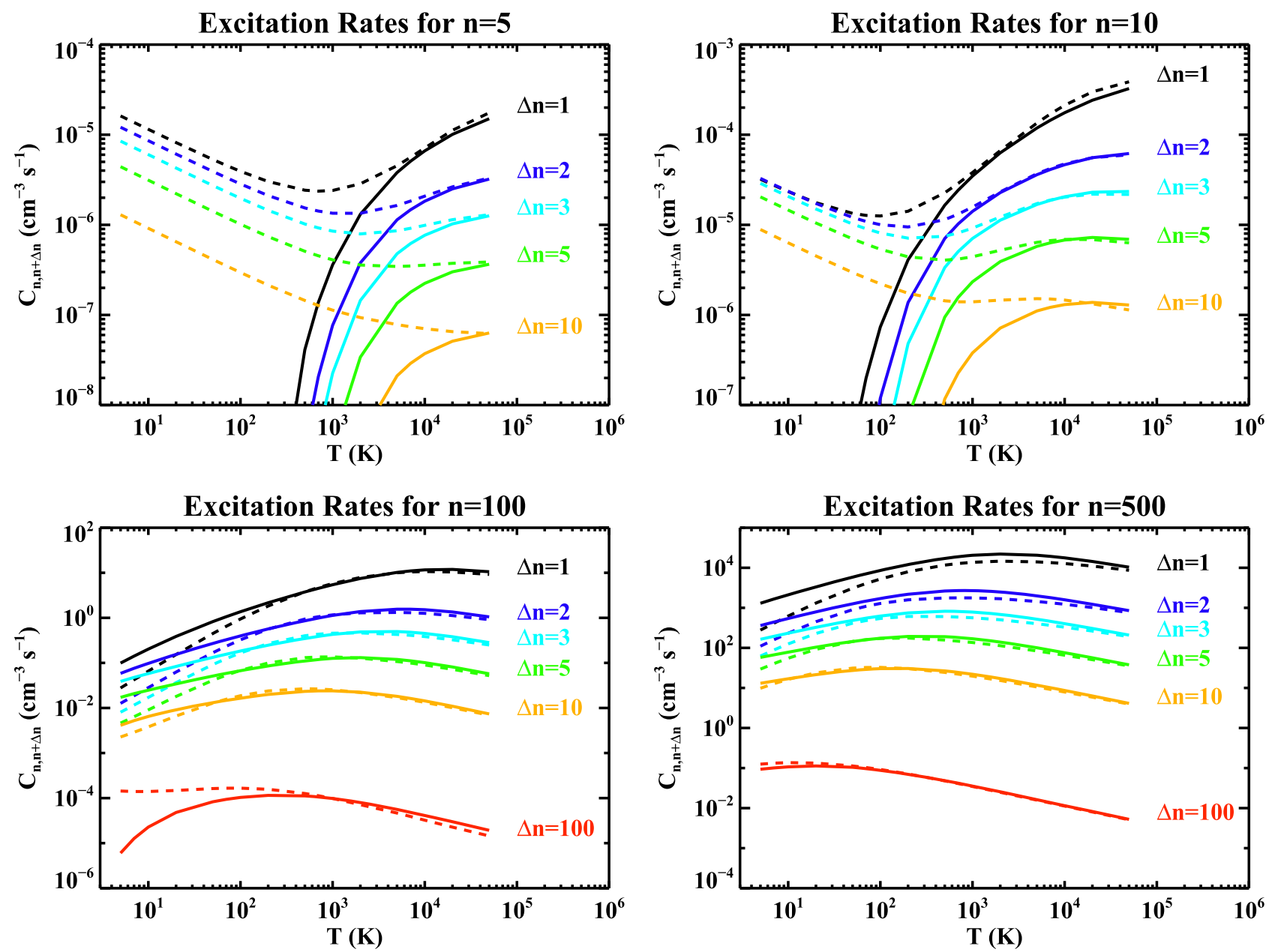

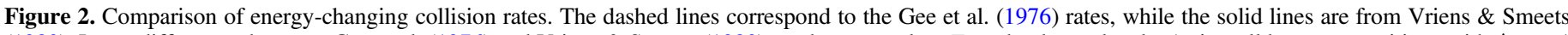

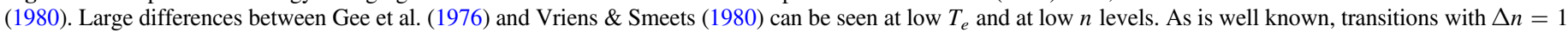
dominate. The difference between $\Delta n>1$ and $\Delta n=1$ rates is less at lower $T_{e}$.

obtain values for the ionization cross section (Appendix D). Values for the radiative recombination $\left(\alpha_{n l}\right)$ coefficients were obtained using the Milne relation and standard formulas (e.g., Rybicki \& Lightman 1986, Appendix D). The program provided by Storey \& Hummer (1991) only produces reliable values up to $n \sim 500$ due to cancellation effects in the iterative procedure. In order to avoid cancellation effects, the values computed here were obtained by working with logarithmic values in the recursion formula. As expected, our values for the rates match those of Storey \& Hummer (1991) well.

For the $n$ method, we require the sum of the individual $\alpha_{n l}$ values:

$$
\alpha_{n}=\sum_{l=0}^{n-1} \alpha_{n l} .
$$

The averaged $\alpha_{n}$ values agree well with the approximated formulation of Seaton (1959a) to better than 5\%, validating our approach.

\subsubsection{Collisional Ionization and Three-body Recombination}

Collisional ionization occurs when an atom encounters an electron and, due to the interaction, a bound electron from the atom is ionized. Schematically the process can be represented as

$$
X_{n}+e^{-} \rightleftarrows X^{+}+e^{-}+e^{-} .
$$

The inverse process is given by the three-body recombination, and the value for the three-body recombination rate is obtained from the detailed balance:

$$
\begin{aligned}
C_{i, n} & =\frac{N_{n}(\mathrm{LTE})}{N_{\text {ion }} N_{e}} C_{n, i}, \\
& =\left(\frac{h^{2}}{2 \pi m_{e} k T e}\right)^{3 / 2} n^{2} e^{\chi_{n}} C_{n, i}, \\
& =\Lambda\left(T_{e}\right)^{3} n^{2} e^{\chi_{n}} C_{n, i} .
\end{aligned}
$$

We used the formulation of Brocklehurst \& Salem (1977) and compared the values with those from the formulation given by Vriens \& Smeets (1980). For levels above 100 and at $T_{e}=10 \mathrm{~K}$, the Brocklehurst \& Salem values are a factor of $\lesssim 2$ larger, but the differences quickly decrease for higher temperatures. To obtain the $C_{n l, i}$ values that are needed in the $n l$ method, we followed Hummer \& Storey (1987) and assumed that the rates are independent of the angular 
momentum. The mathematical formulation is reproduced in Appendix $\mathrm{F}$ for the convenience of the reader.

\subsubsection{Dielectronic Recombination and Autoionization on Carbon Atoms}

The dielectronic recombination process consists of dielectronic capture followed by stabilization. Dielectronic capture involves an electron recombining into a level $n$ while simultaneously exciting one of the bound electrons (left side of Equation (25), below). This state $\left(X_{n}^{*}\right)$ is known as an autoionizing state. In this autoionizing state, the atom can stabilize either by releasing the recombined electron through autoionization (inverse process of dielectronic capture) or through radiative stabilization (right-hand side of Equation (25)). Dielectronic capture and autoionization are only relevant for atoms with more than one electron:

$$
X^{+}+e^{-} \rightleftarrows X_{n l}^{*} \rightarrow X_{n^{\prime} l^{\prime}}+h \nu .
$$

For $\mathrm{C}^{+}$recombination, at $T_{e} \sim 100 \mathrm{~K}$, free electrons in the plasma can recombine to a high $n$ level, and the kinetic energy is transferred to the core of the ion, producing an excitation of the ${ }^{2} P_{1 / 2}-{ }^{2} P_{3 / 2}$ fine structure level of the $\mathrm{C}^{+}$atom core (which has a difference in energy $\Delta E=92 \mathrm{~K}$ ). Due to the long radiative lifetime of the fine structure transition $\left(4 \times 10^{5} \mathrm{~s}\right)$, radiative stabilization can be neglected.

Following Watson et al. (1980), Ponomarev \& Sorochenko (1992) compute the autoionization rate using the formulation by Seaton et al. (1976):

$$
A_{n l}^{a}=4 \frac{R y c}{h} \frac{\Omega(l)}{n^{3} \omega(j, n l)},
$$

with $\Omega(l)$ the collision strength for the ${ }^{2} P_{1 / 2}-{ }^{2} P_{3 / 2}$ excitation at the threshold. As in Watson et al. (1980), we used the formula obtained by Osterbrock (1965):

$$
\Omega(l)=\frac{227}{(2 l-1)(2 l+1)(2 l+3) l(l+2)},
$$

valid for $l>4$. In order to avoid the singularity at $l=0$, we computed the autoionization rate, $A_{n l}^{a}$, from the approximate expression given in Dickinson (1981):

$$
A_{n l}^{a}=2.25 \frac{2 \pi R y c}{n^{3}(l+1 / 2)^{6}},
$$

which is valid for $l>10$. The dielectronic recombination rate is obtained by the detailed balance:

$$
N_{1 / 2}^{+} N_{e} \alpha_{n l}^{d}=N_{n l} A_{n l}^{a} \text {. }
$$

Walmsley \& Watson (1982) defined $b_{d i}$ as the departure coefficient when autoionization/dielectronic recombination dominates:

$$
\begin{aligned}
b_{d i} & =\frac{g_{1 / 2} N_{1 / 2}^{+}}{g_{3 / 2} N_{3 / 2}^{+}} \exp \left[-\Delta E / k T_{e}\right], \\
& =\frac{1}{R} .
\end{aligned}
$$

In this work we are interested in the dielectronic capture and subsequent radiative and collisional redistribution. The cascade calculations necessary to compute the total, level-resolved dielectronic recombination rates are beyond the scope of this work. These rates have been published elsewhere (e.g., Safronova

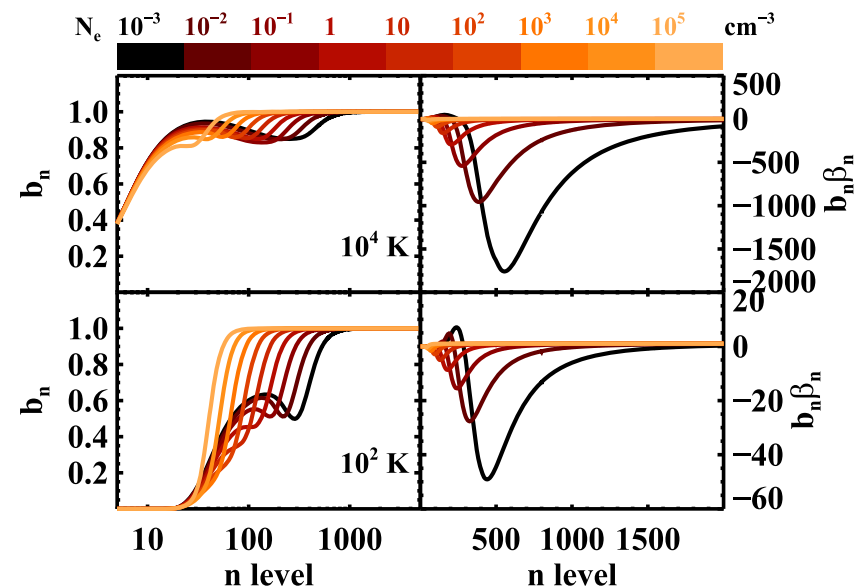

Figure 3. The $b_{n}$ values (left) and $b_{n} \beta_{n}$ values (right) for the hydrogenic approximation at $T_{e}=10^{4}$ and $10^{2} \mathrm{~K}$ (upper and lower panels, respectively) for different densities $\left(N_{e}\right.$, color scale). The departure coefficients obtained using the $n l$ method show a "bump" at low $n$ levels. The strength and position of the "bump" depend on the physical conditions. As density increases, the $l$-changing collisions redistribute the electron population more effectively.

et al. 1998; Altun et al. 2004). However, we note that-for the parameter space of interest to us-these published rates do not depend on the autoionization rates and essentially sum over the relevant Einstein A transition rates. We have verified that our Einstein A coefficients are in agreement with those used by Gordon \& Sorochenko (2009, p. 282) and Storey \& Hummer (1991).

\section{Results}

The behavior of CRRLs with frequency depends on the level population of carbon via the departure coefficients. We compute departure coefficients for carbon atoms by solving the level population equation using the rates described in Section 2.3 and the approach in Section 2.2. Here, we present values for the departure coefficients and provide a comparison with earlier studies in order to illustrate the effect of our improved rates and numerical approach. A detailed analysis of the line strength under different physical conditions relevant for the diffuse clouds and the effects of radiative transfer are provided in an accompanying article (Paper II).

\subsection{Departure Coefficient for Carbon Atoms}

The final departure coefficients for carbon atoms $\left(b_{n}^{\text {final }}\right)$ are obtained by computing the departure coefficients recombining from both parent ions, those in the ${ }^{2} P_{1 / 2}$ level and those in the ${ }^{2} P_{3 / 2}$ level. Therefore, it is illustrative to study the individual departure coefficients for the ${ }^{2} P_{1 / 2}$ core, which are hydrogenic, and the departure coefficients for the ${ }^{2} P_{3 / 2}$ core separately.

\subsubsection{Departure Coefficient in the Hydrogenic Approximation}

In Figure 3 we show example $b_{n}$ and $b_{n} \beta_{n}$ values obtained in the hydrogenic approximation at $T_{e}=10^{2}$ and $10^{4} \mathrm{~K}$ for a large range in density. The behavior of the $b_{n}$ values as a function of $n$ can be understood in terms of the rates that are included in the level population equation. At the highest $n$ levels, collisional ionization and three-body recombination dominate the rates in the level population equation, and the $b_{n}$ values are close to unity. We can see that, as the density increases, collisional equilibrium occurs at lower $n$ levels, and the $b_{n}$ values approach unity at lower levels. In contrast, for the 


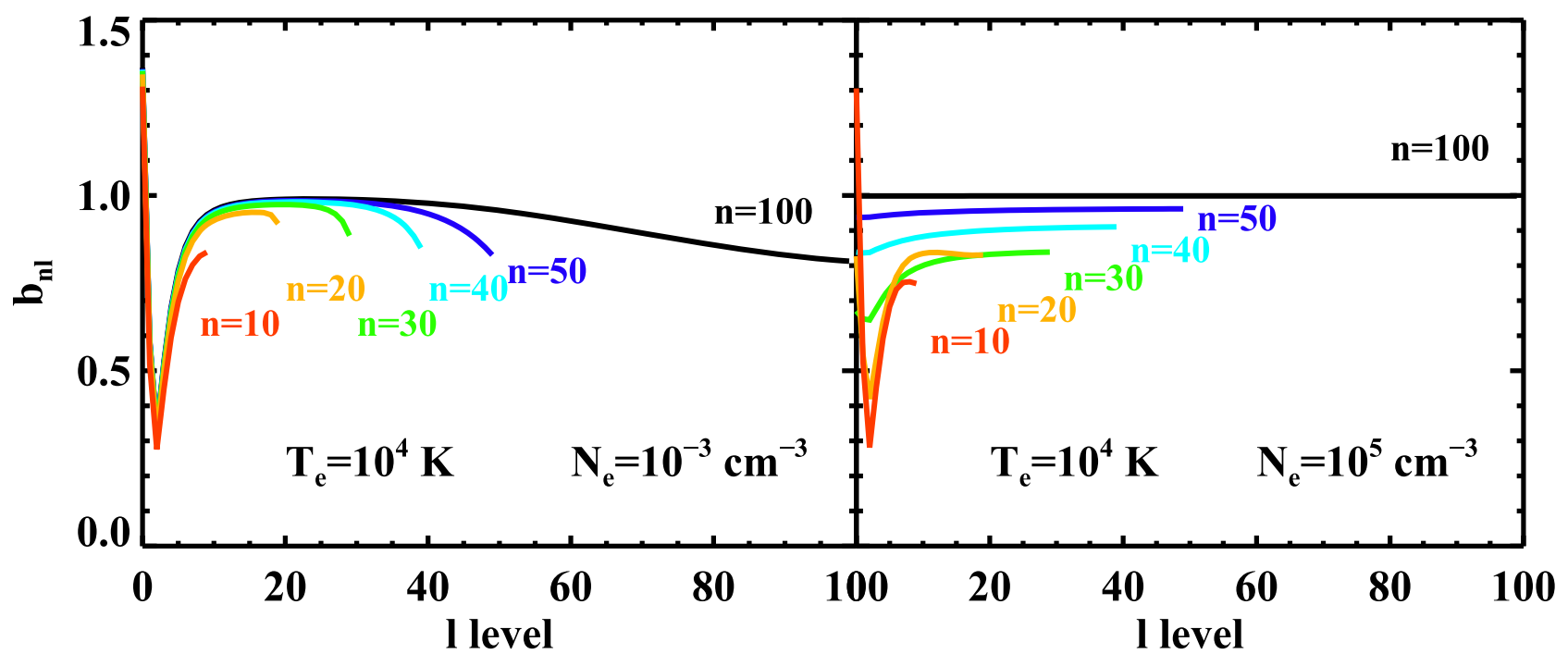

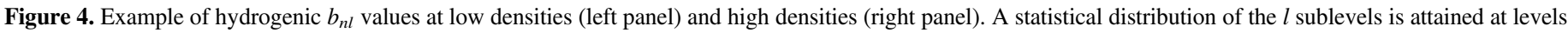

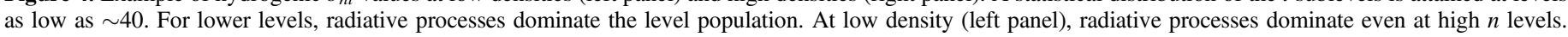

lowest $n$ levels, the level population equation is dominated by radiative processes, and the levels drop out of collisional equilibrium. As the radiative rates increase with decreasing $n$ level, the departure coefficients become smaller. We note that differences in the departure coefficients for the low $n$ levels for different temperatures are due to the radiative recombination rate, which has a $T_{e}^{-3 / 2}$ dependence.

At intermediate $n$ levels, the behavior of the $b_{n}$ as a function of $n$ shows a more complex pattern with a pronounced "bump" in the $b_{n}$ values for intermediate levels ( $n \sim 10$ to 100 ). To guide the discussion, we refer the reader to Figure 3. Starting at the highest $n, b_{n} \rightarrow 1$, as mentioned above. For these high $n$ levels, $l$-changing collisions efficiently redistribute the electron population among the $l$ states and, at high density, the $b_{n l}$ departure coefficients are unity as well (Figure 4, upper panels). For lower values of $n$, the $b_{n}$ values decrease due to an increased importance of spontaneous transitions. At these $n$ levels, the $b_{n}$ values obtained by the $n l$ method differ little from the values obtained by the $n$ method, since $l$-changing collisions efficiently redistribute the electrons among the $l$ sublevels for a given $n$ level. For lower $n$ levels, the effects of considering the $l$ sublevel distribution become important as $l$-changing collisions compete with spontaneous decay, effectively "storing" electrons in high $l$ sublevels for which radiative decay is less important. Specifically, the spontaneous rate out of a given level is approximately $A_{n l} \simeq 10^{10} / n^{3} / l^{2}\left(\mathrm{~s}^{-1}\right)$ and is higher for lower $l$ sublevels. Thus, high $l$ sublevels are depopulated more slowly relative to lower $l$ sublevels on the same $n$ level. This results in a slight increase in the departure coefficients. Reflecting the statistical weight factor in Equation (5), the higher $l$ sublevels dominate the final $b_{n}$ value, resulting in an increase in the final $b_{n}$ value. As the density increases, the $l$ sublevels approach a statistical distribution faster. As a result, the influence of the $l$ sublevel population on the final $b_{n}$ is larger for lower densities than for higher densities at a given $T_{e}$. The interplay of the rates produce the "bump" that is apparent in the $b_{n}$ distribution (Figure 3).

The influence of $l$-changing collisions on the level populations and the resulting increase in the $b_{n}$ values were already presented by Hummer \& Storey (1987) and analyzed in detail by Strelnitski et al. (1996) in the context of hydrogen masers. The results of our level population models are in good agreement with those provided by Hummer \& Storey (1987), as we show in Section 3.2.

\subsubsection{Departure Coefficient for Carbon Atoms including Dielectronic Capture}

Only carbon atoms recombining to the ${ }^{2} P_{3 / 2}$ ion core are affected by dielectronic capture. Having analyzed the departure coefficients for the hydrogenic case, we focus now on the $b_{n l}^{3 / 2}$ values and the resulting $b_{n}^{\text {final }}$ as introduced in Section 2.1.

Figure 5 shows example values for $b_{n}^{3 / 2}$ for $T_{e}=50,100$, 200 , and $1000 \mathrm{~K}$ and electron densities between $10^{-3}$ and $10^{2} \mathrm{~cm}^{-3}$. As pointed out by Watson et al. (1980), the low-lying $l$ sublevels are dominated by the dielectronic process, and the $b_{n l}^{3 / 2}$ values are equal to $b_{d i}$ (Equation (30)). As can be seen in Figure 1, such values can be much larger than unity at low densities, resulting in an overpopulation of the low $n$ levels for the $3 / 2$ ion cores. In Figure 6 we show $b_{n}^{\text {final }}$ as a function of $n$ level under the same conditions. We see that at high electron densities the departure coefficients show a behavior similar to the hydrogenic values. Furthermore, an increase in the level population to values larger than unity is seen at low densities and moderate to high temperatures.

To guide the discussion, we analyze the behavior of the $b_{n}^{\text {final }}$ when autoionization/dielectronic capture dominates. This occurs at different levels depending on the values of $T_{e}$ and $N_{e}$ considered. Nevertheless, it is instructive to understand the behavior of the level population in extreme cases. When autoionization/dielectronic capture dominates, the $b_{n}^{\text {final }}$ in Equation (12) is given by

$$
b_{n}^{\text {final }} \approx \frac{b_{n}^{1 / 2}+\left[N_{3 / 2}^{+} / N_{1 / 2}^{+}\right]_{\mathrm{LTE}}}{1+R\left[N_{3 / 2}^{+} / N_{1 / 2}^{+}\right]_{\mathrm{LTE}}} .
$$

At high densities, $R$ approaches unity, and we note two cases. The first case is when $T_{e}$ is high, the maximum value of $\left[N_{3 / 2}^{+} / N_{1 / 2}^{+}\right]_{\text {LTE }}=2$, meaning that a large fraction of the ions are in the ${ }^{2} P_{3 / 2}$ core. Consequently, $b_{n}^{\text {final }} \approx\left(b_{n}^{1 / 2}+2\right) / 3$, so the effect of dielectronic capture is to increase the level population as compared to the hydrogenic case. We also note 


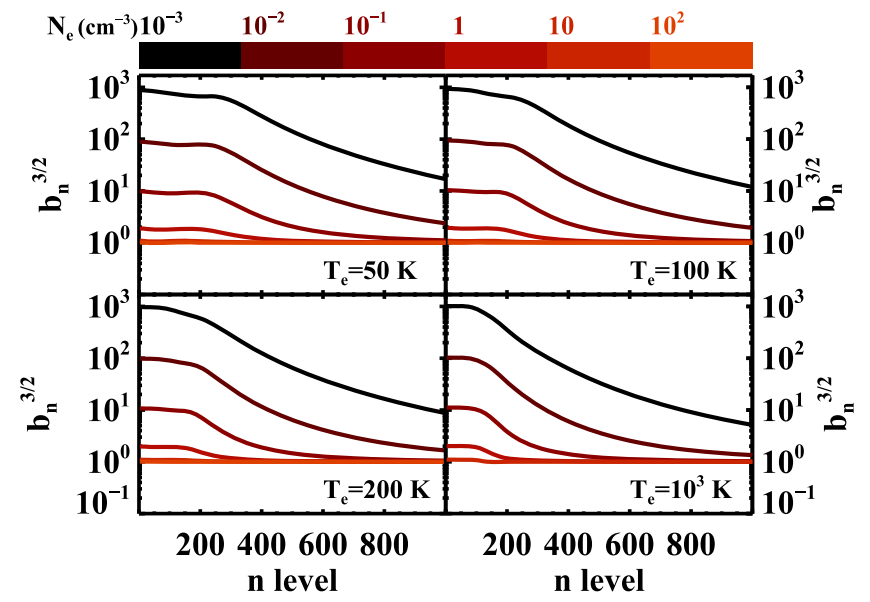

Figure 5. Departure coefficients for the ${ }^{2} P_{3} / 2$ parent ions as a function of $n$ at $T_{e}=50,100,200$, and $1000 \mathrm{~K}$ for different densities $\left(N_{e}\right.$, color scale). The values for low $n$ levels are close to $b_{d i}$ and decrease toward a value of one. At high densities, $b_{n}^{3 / 2} \approx 1$.

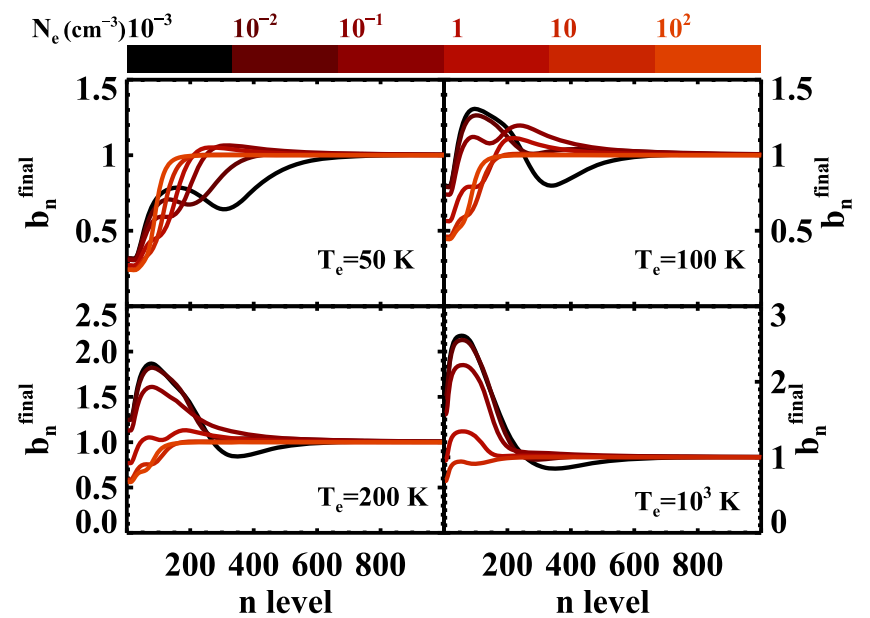

Figure 6. Final departure coefficients for carbon atoms $\left(b_{n}^{\text {final }}\right)$ as a function of $n$ level at $T_{e}=50,100,200$, and $1000 \mathrm{~K}$ for different densities $\left(N_{e}\right.$, color scale). The "bump" seen in hydrogenic atoms is amplified by dielectronic capture. As density increases, the $b_{n}^{\text {final }}$ values are closer to the hydrogenic value.

that since $b_{n}^{1 / 2} \leqslant 1$, the final $b_{n}^{\text {final }} \leqslant 1$. The second case we analyze is for low $T_{e}$, where the ion LTE ratio is low and most of the ions are in the ${ }^{2} P_{1 / 2}$ core. Thus, $b_{n}^{\text {final }} \approx b_{n}^{1 / 2}$ and the departure coefficients are close to hydrogenic.

At low densities, $R \ll 1$ and, as above, we study two cases. The first is when $T_{e}$ is high, the maximum value of $\left[N_{3 / 2}^{+} / N_{1 / 2}^{+}\right]_{\text {LTE }}=2$ and $b_{n}^{\text {final }} \approx b_{n}^{1 / 2}+2$, so dielectronic capture produces a large overpopulation as compared to the hydrogenic case. The second case is when $T_{e}$ is low and most of the ions are in the ${ }^{2} P_{1 / 2}$ level and, as in the high-density case, the $b_{n}^{\text {final }} \approx b_{n}^{1 / 2}$. We note from this analysis that overpopulation of the $b_{n}^{\text {final }}$ (relative to the hydrogenic case) is only possible for a range of temperatures and densities. In particular, $b_{n}^{\text {final }}$ is maximum for high temperatures and low densities.

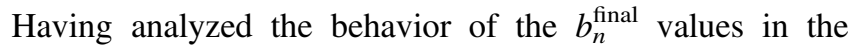
extreme $b_{n}^{3 / 2}=b_{d i}$ case, now we analyze the behavior of $b_{n}^{3 / 2}$ with $n$. The population in the low $n$ levels is dominated by

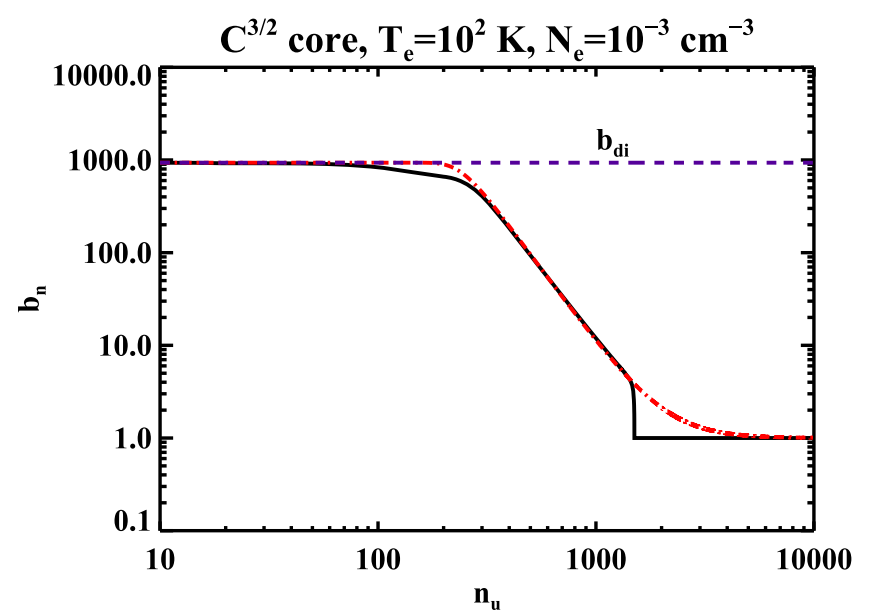

Figure 7. The $b_{n}^{3 / 2}$ values for carbon as a solid black line; the discontinuity at $n=1500$ is due to the $n_{\text {crit }}$ value. Overplotted as a red dot-dashed line is the approximation in Equation (32). The blue dashed line is the value of $b_{d i}$.

dielectronic capture (Watson et al. 1980; Walmsley \& Watson 1982) and $b_{n}^{3 / 2}=b_{d i}$ up until a certain $n$ level where $b_{n}^{3 / 2}$ begins to decrease down to a value of one. The $n$ value where this change happens depends on temperature, moving to higher $n$ levels as $T_{e}$ decreases. To understand this further, we analyze the rates involved in the $l$ sublevel population (Figure 5). The low $l$ sublevels are dominated by dielectronic capture and autoionization, and the $b_{n l}$ values for the $3 / 2$ ion cores are $b_{n l}^{3 / 2}=b_{d i}$. For the higher $l$ sublevels, other processes (mainly collisions) populate or depopulate electrons from the level $n$, and the net rate is lower than that of the low $l$ dielectronic capture/autoionization. This lowers the $b_{n l}$ value, which is effectively delayed by $l$-changing collisions since they redistribute the population of electrons in the $n$ level. The $b_{n l}$ for the highest $l$ values dominate the value of $b_{n}^{3 / 2}$ due to the statistical weight factor.

We note that the behavior of the $b_{n}^{3 / 2}$ cores as a function of $n$ (see Figure 7) can be approximated by

$$
b_{n}^{3 / 2} \approx \tanh \left(\left[\frac{l_{m}}{n}\right]^{3}\right) \times\left(b_{d i}-1\right)+1
$$

with $b_{d i}$ defined as in Walmsley \& Watson (1982) (Equation (30)), and $l_{m}$ was derived from fitting our results:

$$
l_{m} \approx 60 \times\left(\frac{N_{e}}{10}\right)^{-0.02}\left(\frac{T_{e}}{10^{4}}\right)^{-0.25} .
$$

In diffuse clouds, the integrated line-to-continuum ratio is proportional to $b_{n} \beta_{n}$. We note that the $\beta_{n}$ behavior is more complex, as can be seen in Figure 8. The low $n$ "bump" on the $b_{n}^{\text {final }}$ makes the $b_{n} \beta_{n}$ high at low densities and for levels between about 150 and 300. Since the $b_{n}^{\text {final }}$ values decrease from values larger than one to approximately one, the $\beta_{n}$ changes sign. In Figure 9 we show the electron density as a function of the level where the change of sign on the $b_{n} \beta_{n}$ occurs. At temperatures higher than about 200, our models for $N_{e}=0.1 \mathrm{~cm}^{-3}$ show no change of sign due to the combined effects of $l$-changing collisions and dielectronic capture. 


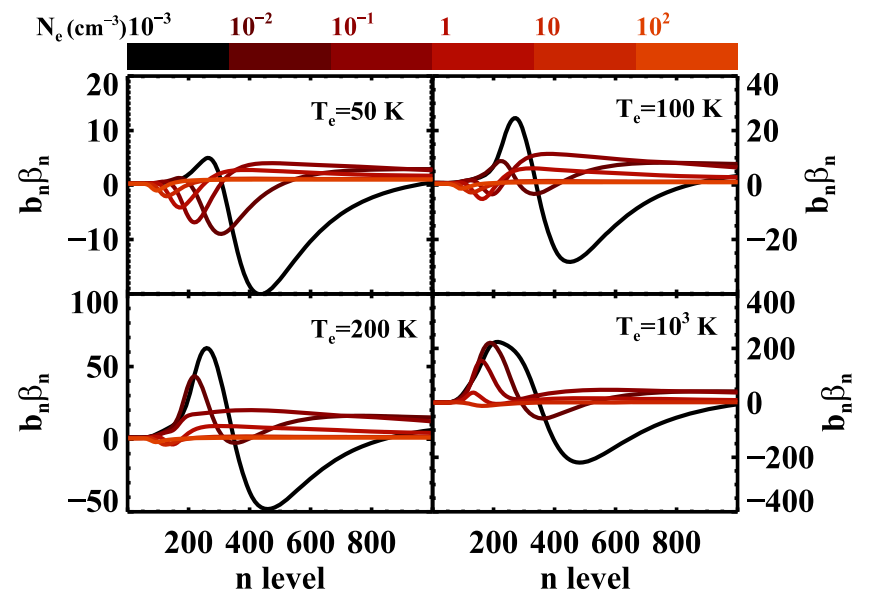

Figure 8. The $b_{n} \beta_{n}$ values for carbon atoms at $T_{e}=50,100,200$, and $1000 \mathrm{~K}$ for different densities ( $N_{e}$, color scale).

\subsection{Comparison with Previous Models}

The level population of hydrogenic atoms is a well-studied problem. Here, we will describe the effects of the updated collision rates as well as point out differences due to the improved numerical method.

\subsubsection{Hydrogenic Atoms}

At the lowest densities, we can compare our results for hydrogenic atoms with the values of Martin (1988) for hydrogen atoms. The results of Martin (1988) were obtained in the low-density limit: no collision processes were taken into account in his computations. The results are given in terms of the emissivity of the line normalized by the $\mathrm{H} \beta$ emissivity. As can be seen in Figure 10, our results agree to better than $5 \%$ and for most levels to better than $0.5 \%$.

At high densities, we compare the hydrogenic results obtained here with those of Hummer \& Storey (1987). Our approach reproduces well the $b_{n l}$ (and $b_{n}$ ) values of Hummer \& Storey (1987) (to better than 1\%) when using the same collision rates (Pengelly \& Seaton 1964; Gee et al. 1976), as can be seen in Figure 11. We note that the effect of using different energy-changing rates $\left(C_{n, n^{\prime}}\right)$ has virtually no effect on the final $b_{n}$ values. On the other hand, using Vrinceanu et al. (2012) values for the $C_{n l, n l \pm 1}$ rates results in differences in the $b_{n}$ values of $30 \%$ at $T_{e}=10^{3} \mathrm{~K}, N_{e}=100 \mathrm{~cm}^{-3}$. As expected, the difference is less at higher temperatures and densities since the values are closer to equilibrium (see Figure 12). At low $n$ levels, our results for high $l$ levels are overpopulated as compared to the values of Hummer \& Storey (1987), leading to an increase in the $b_{n}$ values.

\subsubsection{Carbon}

Now we compare departure coefficients obtained here with the results of Ponomarev \& Sorochenko (1992) and the effect of including $l$-changing collisions on the departure coefficients; see Figures 13 and 14. It is worth mentioning that Ponomarev \& Sorochenko (1992) did not include $l$-changing collisions and instead assumed a statistical population. We will focus the discussion on the $b_{n}$ values from Ponomarev \& Sorochenko (1992) as the Walmsley \& Watson (1982) values are similar.

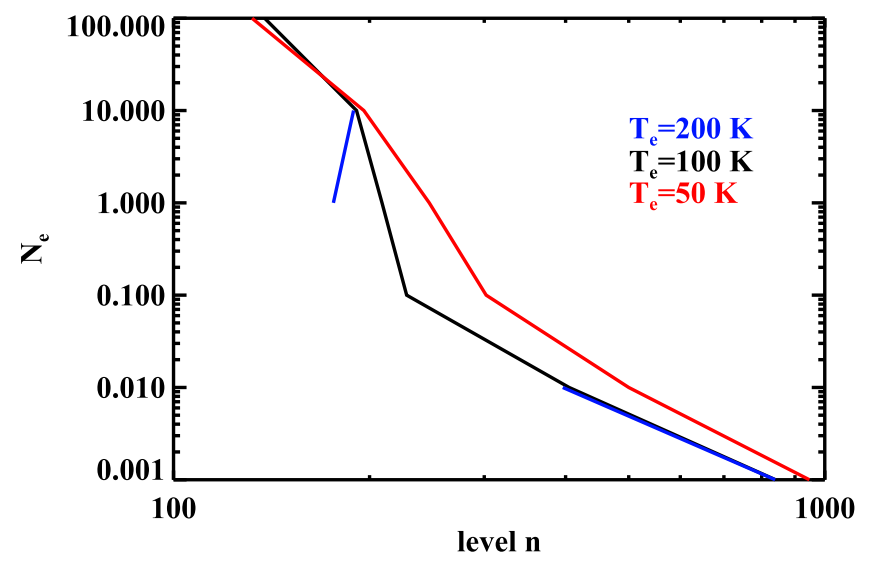

Figure 9. Levels where the $b_{n} \beta_{n}$ values go to zero for $T_{e}=50$, 100 , and $200 \mathrm{~K}$. At temperatures larger than $200 \mathrm{~K}$ and for electron densities around $10^{-1} \mathrm{~cm}^{-3}$, the $b_{n} \beta_{n}$ values do not go through zero.

While the results presented here are remarkably different from those of Walmsley \& Watson (1982) and Ponomarev \& Sorochenko (1992), some trends are similar. We will first discuss the differences. Our results in Figures 13 and 14 show a pronounced "bump" for low $n$ in the range 50-150. This bump is similar to what we see for the hydrogenic approximation but enhanced by dielectronic capture (see Figures 3.6 and 8; Section 3.1.2). As discussed in Section 3.1.1, this bump arises at these intermediate $n$ levels because collisions compete with spontaneous decay, effectively "storing" electrons in high $l$ sublevels for which radiative decay is less important. This means that the inclusion of $l$-changing collisions leads to significantly larger $b_{n}$ values for $n$ in the range 50-150 as compared to Ponomarev \& Sorochenko (1992). Regardless of the $l$-changing collision rates used, at higher $n$ we note that our $b_{n}$ values with increasing $n$ asymptotically approach unity much faster than for Ponomarev \& Sorochenko (1992). This is especially true for lower electron densities $\left(n_{e}<1.0 \mathrm{~cm}^{-3}\right)$ and is a direct consequence of using the $n l$ method to compute the departure coefficients.

Although the detailed behavior of our $b_{n}$ values differs strongly from Ponomarev \& Sorochenko (1992), there are also similarities in the general trends that we observe as a function of electron density and temperature. In particular, the very low and very high $n$ asymptotic behavior of the $b_{n}$ values is similar to that of Ponomarev \& Sorochenko (1992) in that the highest electron densities for a given electron temperature have the lowest $b_{n}$ values at low $n$ and approach equilibrium $\left(b_{n}=1\right)$ the fastest with increasing $n$. For higher electron densities and lower electron temperatures, our results become increasingly similar to the hydrogenic case and agree with that of Ponomarev \& Sorochenko (1992). This is expected because, as discussed in Section 3.1, at high densities the $b_{n}$ values approach equilibrium.

In terms of $b_{n} \beta_{n}$ our results show, as expected, good agreement with the hydrogenic case and with Ponomarev \& Sorochenko (1992) in the high-density and low-temperature limit. However, for the lower densities and higher temperatures shown in Figures 13 and 14, our models predict $b_{n} \beta_{n}$ values that are lower by up to about an order of magnitude than that of Ponomarev \& Sorochenko (1992). This is particularly striking for the $T_{e}=100 \mathrm{~K}$ and $n_{e}=0.05 \mathrm{~cm}^{-3}$ model shown in Figure 14, where we find that both the maximum negative 

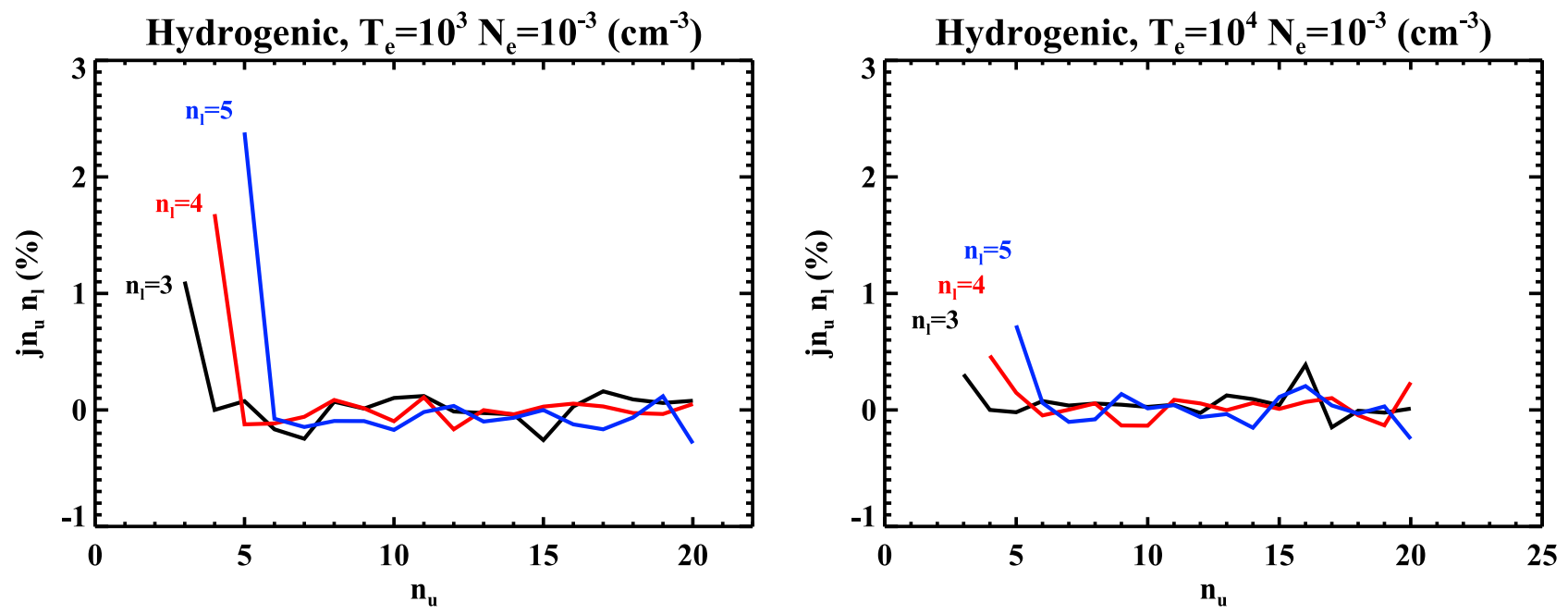

Figure 10. Difference between the emissivities (normalized to $\mathrm{H} \beta$ ) for low $n l$ lines at low density and the results from Martin (1988) in the low- $N_{e}$ approximation. Our results agree to better than $1 \%$ at most levels.
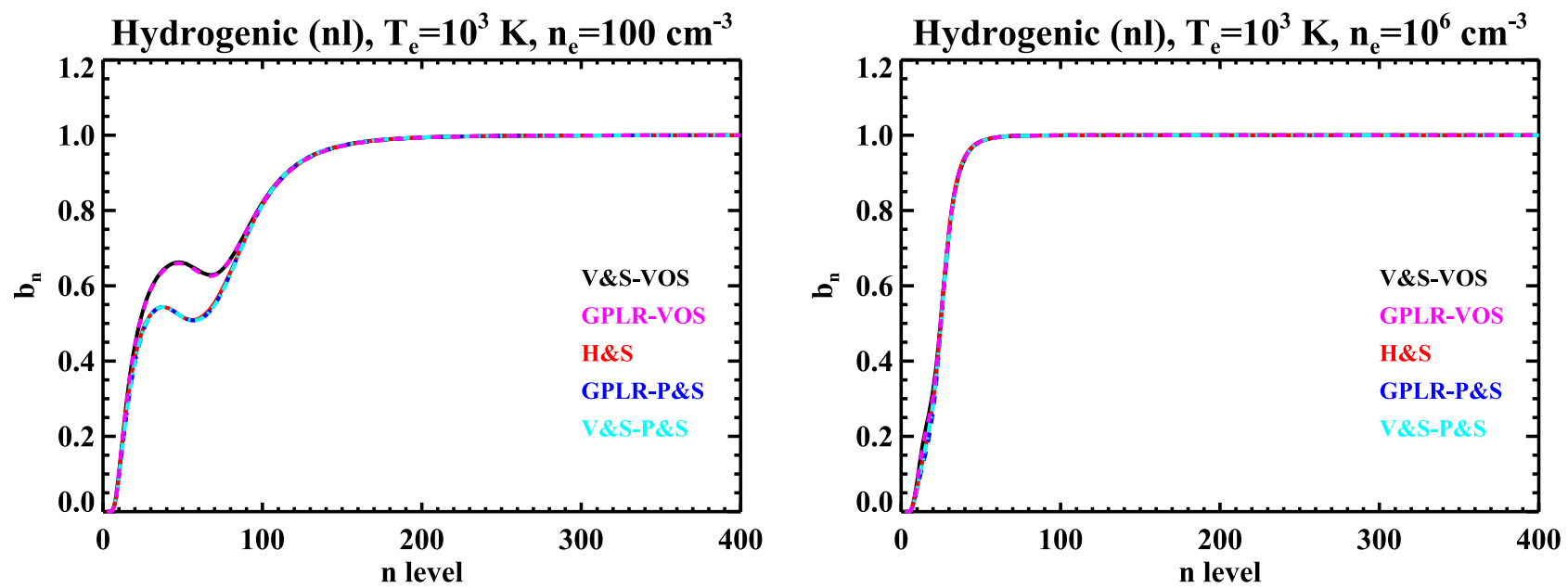

Figure 11. A comparison of the effect of different collision rates on the final $b_{n}$ values for $T_{e}=1000 \mathrm{~K}$ and $N_{e}=100$ and $10^{6} \mathrm{~cm}^{-3}$. H\&S are the departure coefficients from Hummer \& Storey (1987), who used Gee et al. (1976); GPLR corresponds to the use of $n$-changing collision rates from Gee et al. (1976), and V\&S from Vriens \& Smeets (1980); P\&S corresponds to the use of $l$-changing collision rates from Pengelly \& Seaton (1964), and VOS corresponds to Vrinceanu et al. (2012). The largest differences are $\sim 30 \%$ due to the use of different $l$-changing collision rates.

$b_{n} \beta_{n}$ value and the maximum positive $b_{n} \beta_{n}$ value are more than an order of magnitude lower than the corresponding Ponomarev \& Sorochenko (1992) values.

Since the integrated optical depth is directly proportional to the value of $b_{n} \beta_{n}$ (e.g., Shaver 1975; Walmsley \& Watson 1982; Salgado et al. 2017), we can interpret $b_{n} \beta_{n}$ as a stimulation factor. This means that, for a given set of physical conditions, our models predict much lower maximum integrated optical depths for carbon as compared to earlier investigations (e.g., Walmsley \& Watson 1982; Ponomarev $\&$ Sorochenko 1992). This is true for both emission (negative $b_{n} \beta_{n}$ ) and absorption (positive $b_{n} \beta_{n}$ ). In particular, our models predict that equilibrium will be reached at much lower $n$ (typically around $n=600$ ) and thus that the integrated optical at high $n$ (low frequencies) will show a rather flat behavior for $n>600$, whereas the previous models by Walmsley \& Watson (1982) and Ponomarev \& Sorochenko (1992) predict a strong increase with increasing $n$.

We find that, although our $b_{n}$ values asymptotically approach equilibrium at high $n$, this value is not yet reached at $n=1000$.

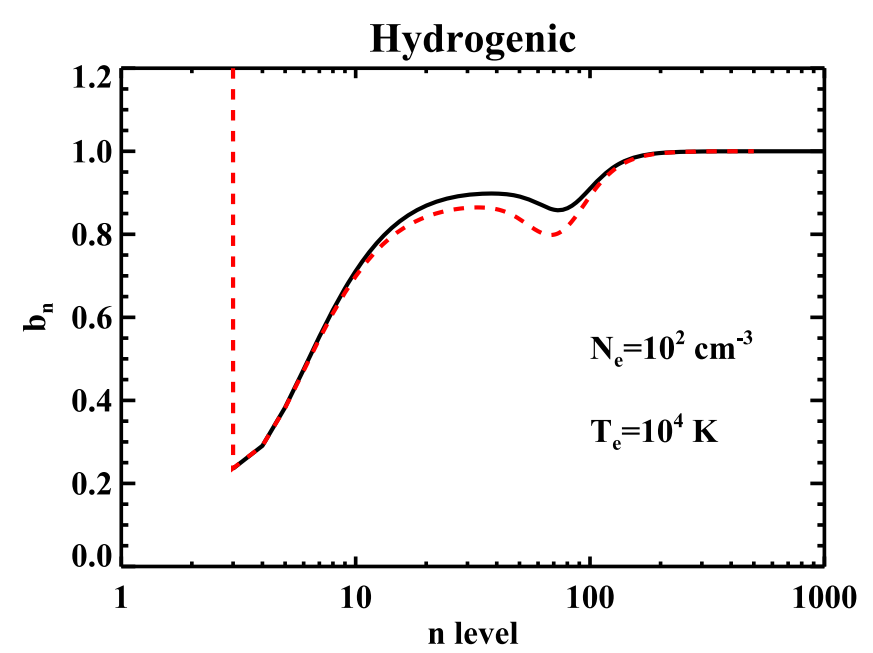

Figure 12. Comparison between our $b_{n}$ values (black) at $T_{e}=10^{4} \mathrm{~K}$ and $N_{e}=100 \mathrm{~cm}^{-3}$ and the results from Hummer \& Storey (1992) (dashed red line). Differences are due to the use of $l$-changing collision rates from Vrinceanu et al. (2012). 

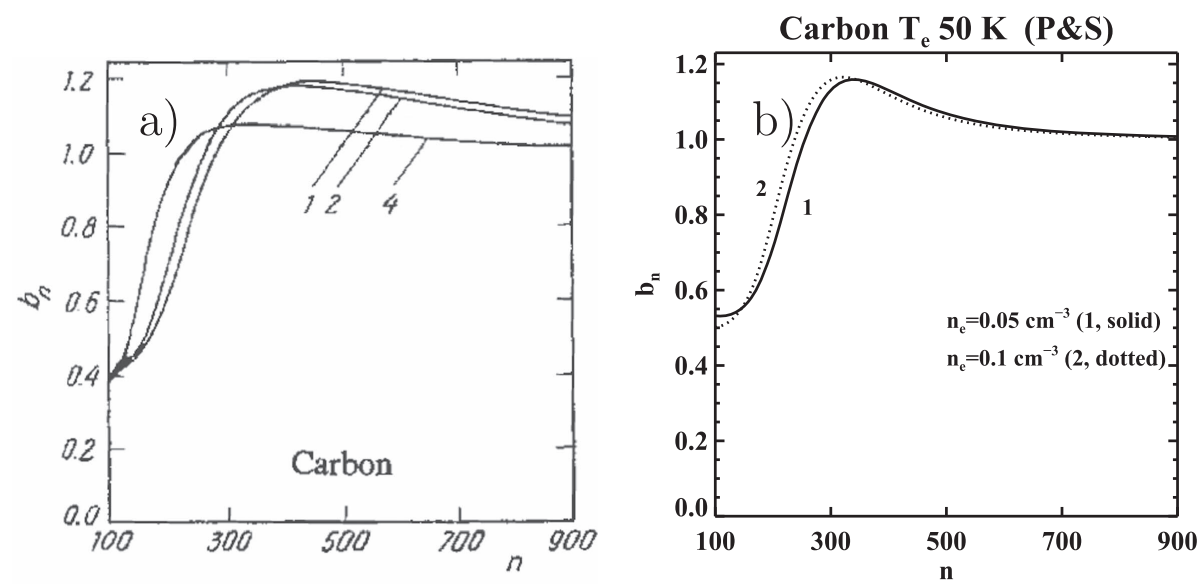

Carbon $T_{\mathrm{e}} 50 \mathrm{~K}$ (P\&S)
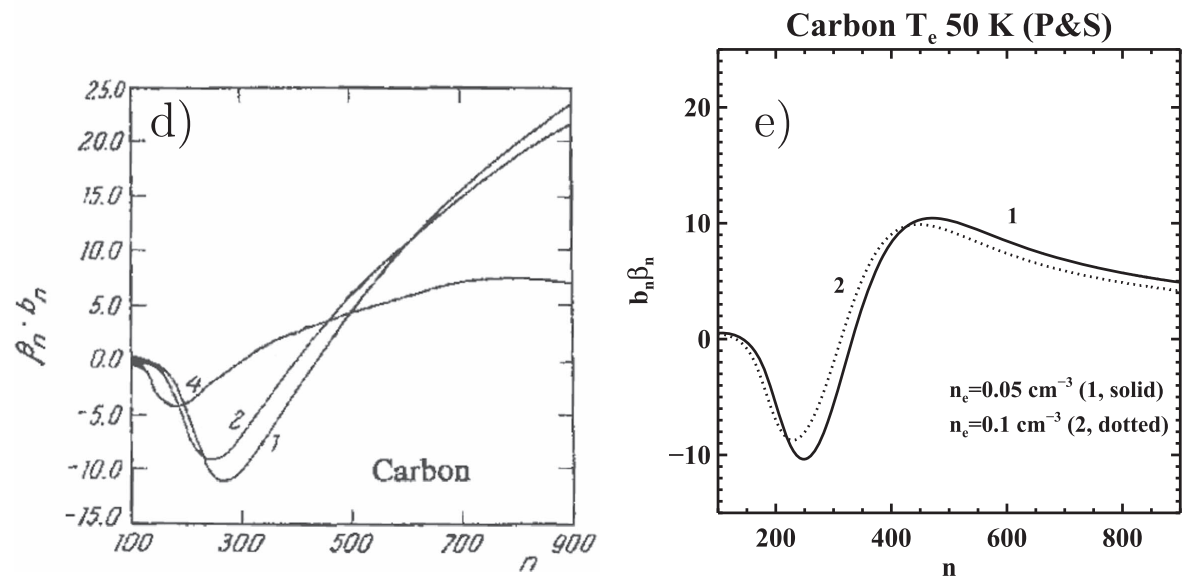

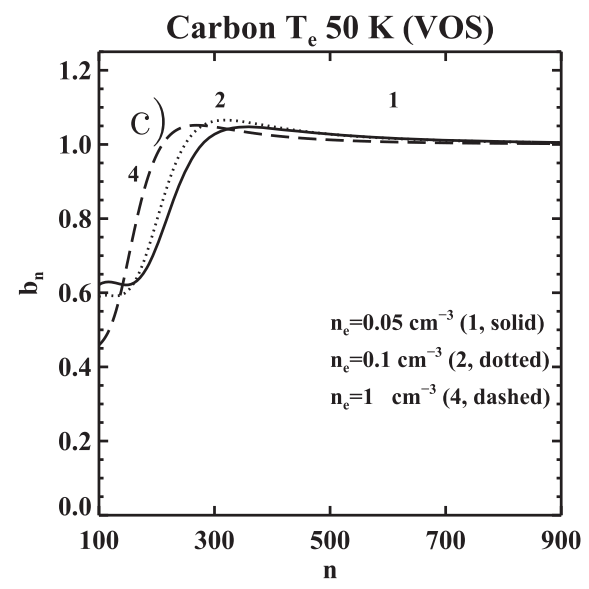

Carbon $T_{e} 50$ K (VOS)

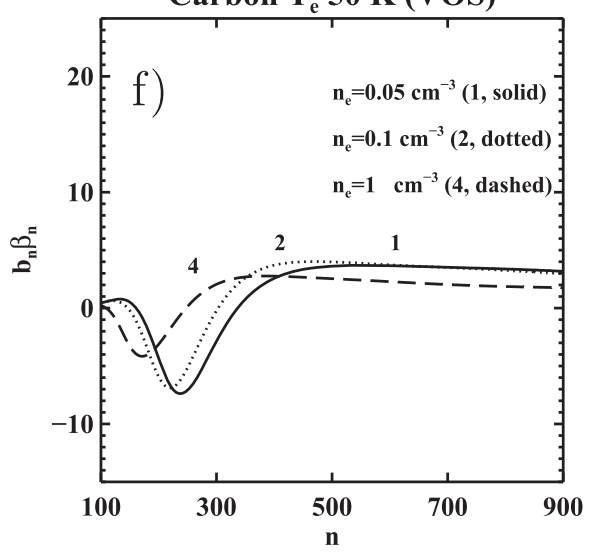

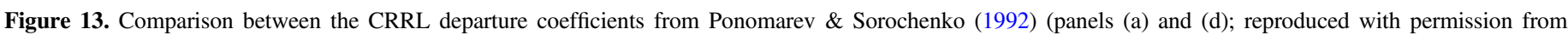

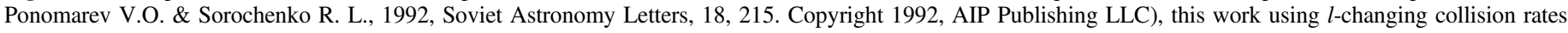

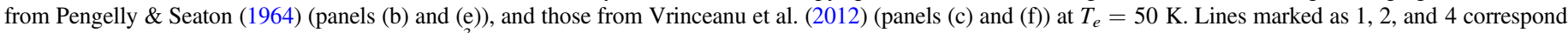

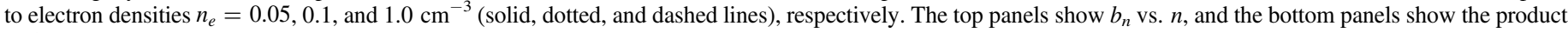
$b_{n} \beta_{n}$ vs. $n$.

Therefore, the $b_{n} \beta_{n}$ values we find are nearly, but not yet completely, constant in the range $n=600-1000$, and as such the dependence of integrated optical depth on $b_{n} \beta_{n}$ remains important at high $n$. Finally, we note that, for sufficiently high electron temperatures and low electron densities, our models predict the existence of a region at intermediate $n(n=100-200)$ where the $b_{n} \beta_{n}$ values can become positive. This behavior is a direct consequence of the inclusion of $l$-changing collisions in our models. A more detailed comparison of the departure coefficients obtained using the $l$-changing collision from Pengelly \& Seaton (1964) and those using the rates from Vrinceanu et al. (2012) (Figure 15) reveals differences of less than $30 \%$ for the conditions of interest for CRRL studies.

Apart from the $l$-changing collisions, there are other potentially important differences between our models and those published by Ponomarev \& Sorochenko (1992). Ponomarev \& Sorochenko (1992) do not provide the explicit values of the rates that they use. However, they refer back to Walmsley \& Watson (1982) for these rates, and as we use the same formalism, we do not think that the rates are at the heart of the discrepancy. In addition, we note that we use somewhat different collision rates in our simulations. However, as illustrated in Figures 13 and 14, the exact collision rates have only limited influence on the $b_{n}$ values. Rather, we suspect that the approximate way the statistical equilibrium equations are solved by Ponomarev \& Sorochenko (1992) may have influenced their results and that including $l$-changing collisions properly rather than adopting a statistical population as done by Ponomarev \& Sorochenko (1992) is important.

A further assessment of the effect of any uncertainty in the adopted dielectronic capture rates on the final departure coefficients can be performed by arbitrarily multiplying the dielectronic capture rate by a factor. We note that a dielectronic capture rate a factor of $30 \%$ higher (lower) increases (decreases) the departure coefficients at low levels $(n<100)$ by $30 \%$. At the higher levels of interest for the study of CRRLs $(n>250)$, a factor of $30 \%$ on the dielectronic capture rates changes the values of the departure coefficients by less than $10 \%$ (Figure 16, upper panels). As expected, the values for $b_{n} \beta_{n}$ are affected more by the change on the dielectronic capture rate and can be altered by factors of a few (Figure 16, lower panels). It is clear that a quantitative interpretation of CRRLs would be served by more accurate dielectronic capture rates that include the fine structure levels.

\section{Summary and Conclusions}

We have solved the level population equation for hydrogenic atoms using novel rates involved in the process. The level population equation is solved in two approximations: the 

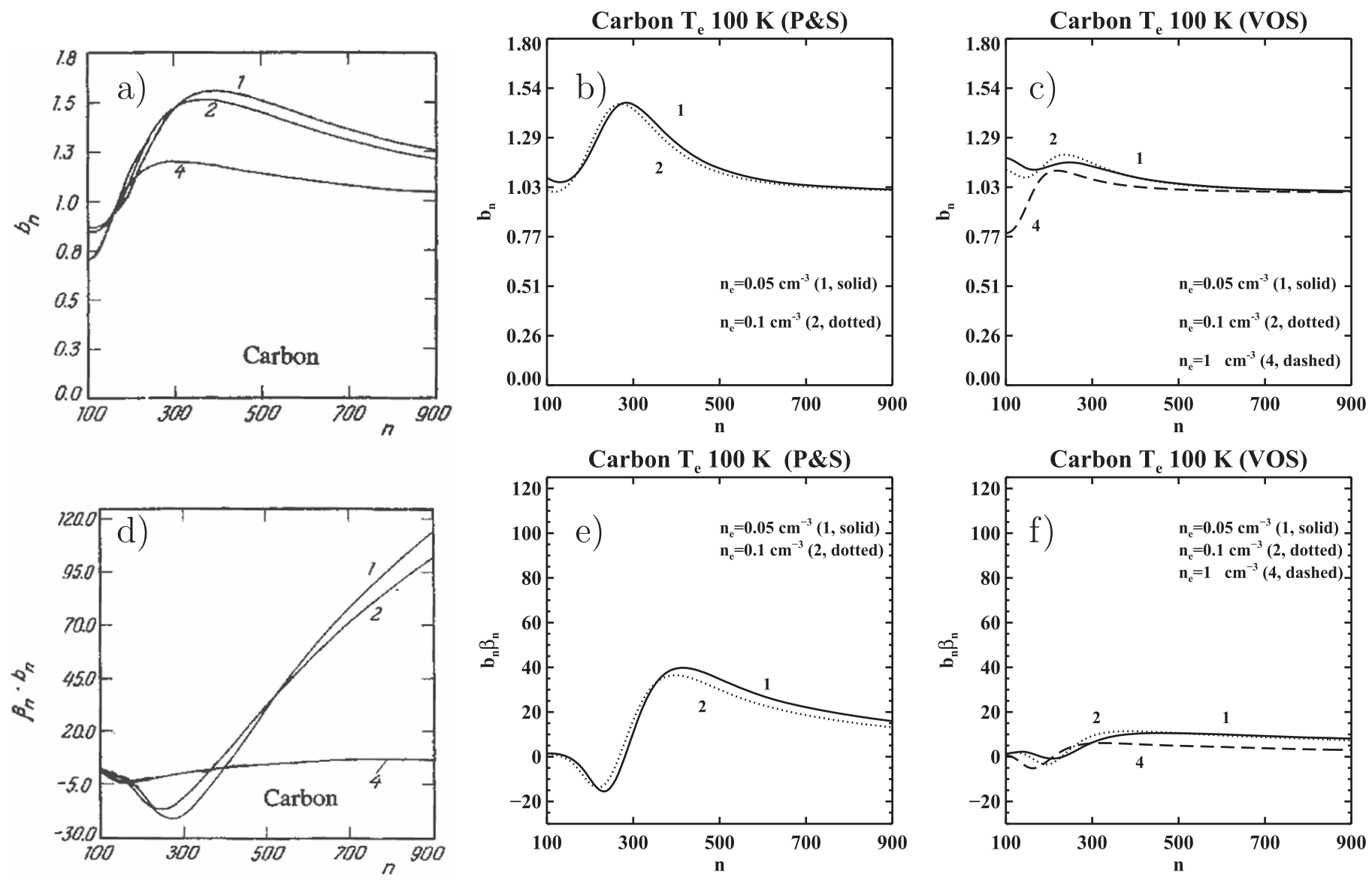

Figure 14. Comparison between the CRRL departure coefficients from Ponomarev \& Sorochenko (1992) (panels (a) and (d); reproduced with permission from Ponomarev V.O. \& Sorochenko R. L., 1992, Soviet Astronomy Letters, 18, 215. Copyright 1992, AIP Publishing LLC), this work using $l$-changing collision rates from Pengelly \& Seaton (1964) (panels (b) and (e)), and those from Vrinceanu et al. (2012) (panels (c) and (f)) at $T_{e}=100 \mathrm{~K}$. Lines marked as 1, 2, and 4 correspond to electron densities $n_{e}=0.05,0.1$, and $1.0 \mathrm{~cm}^{-3}$ (solid, dotted, and dashed lines), respectively. The top panels show $b_{n}$ vs. $n$, and the bottom panels show the product $b_{n} \beta_{n}$ vs. $n$.
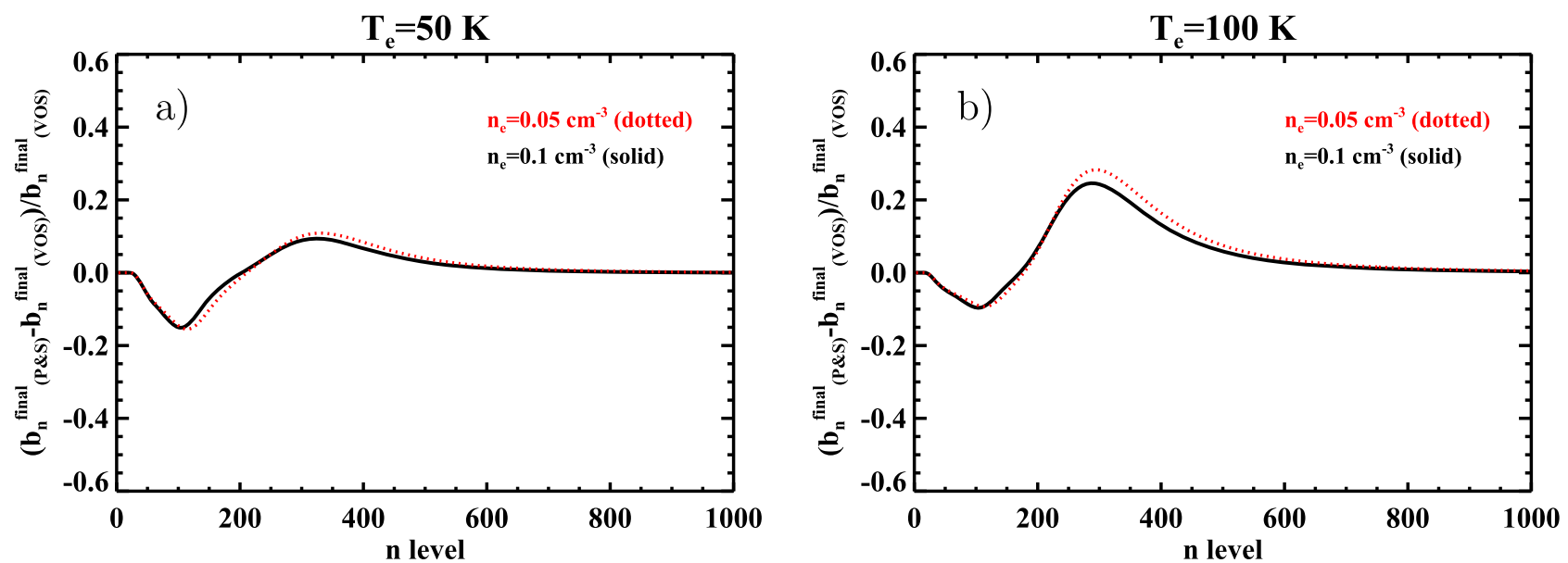

Figure 15. Comparison between the CRRL departure coefficients obtained using l-changing collision rates from Vrinceanu et al. (2012; using their semiclassical result) and Pengelly \& Seaton (1964) at 50 and $100 \mathrm{~K}$. The largest differences are $\sim 30 \%$ at levels $\sim 300$.

$n$ method and the $n l$ method. The departure coefficients obtained using the $n$ method are similar to values from the literature (e.g., Brocklehurst 1970 and Shaver 1975). Our results using the $n l$ method reproduce those from Hummer \& Storey (1987) well, once allowance is made for updates in the collision rates.
By including the dielectronic capture process together with the $\mathrm{nl}$ method, we are able to model the level population of carbon in terms of the departure coefficients. Our results are qualitatively similar to those of Watson et al. (1980) and Walmsley \& Watson (1982). However, the values obtained here differ considerably from those from the literature. The differences can be understood 


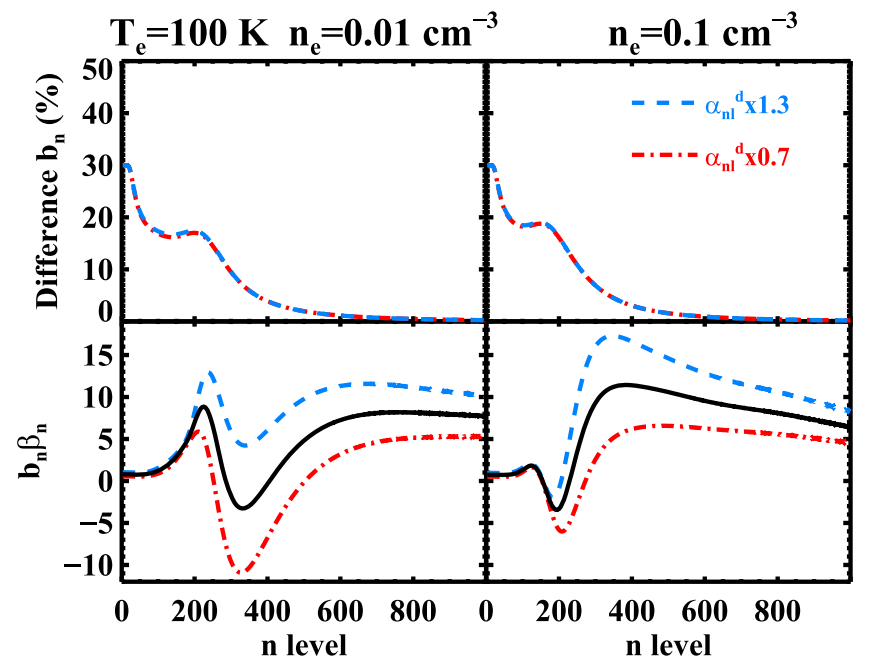

Figure 16. Upper panels: the difference (in percent) of the departure coefficients after multiplying the dielectronic capture rate by a factor of 1.3 (0.7) in blue (red) for a temperature of $100 \mathrm{~K}$ and densities of $0.01 \mathrm{~cm}^{-3}$ (left) and $0.1 \mathrm{~cm}^{-3}$ (right). Lower panels: $b_{n} \beta_{n}$ for the same physical conditions as in the upper panels. At $n$ levels lower than $\sim 200$, the $b_{n} \beta_{n}$ values derived by using the modified dielectronic capture rates (blue and red) are similar to those without modification (black). At higher levels the overall trends are similar, but they can differ by factors of a few.

in terms of the use of improved collision rates and the improved numerical approach using the $n l$ method. We confirm that dielectronic recombination can indeed produce an increase in the values of the departure coefficients at high $n$ levels compared to the hydrogenic values. In particular, the inclusion of $l$-changing collisions is important in calculating the departure coefficients. The exact $l$-changing collision rates are not very well known and are a topic of debate within the atomic physics community (e.g., Guzmán et al. 2016). The semiclassical results by Vrinceanu et al. (2012), used here, differ by up to a factor of $\sim 6$ from their quantum mechanical results, which are closer to the rates presented by Pengelly \& Seaton (1964). The Pengelly \& Seaton (1964) rates are not appropriate for this work as the rates become negative for $n_{e}<0.05 \mathrm{~cm}^{-3}$. We are aware that Guzmán et al. (2017) have very recently presented new $l$-changing rates that may resolve this issue, and we will investigate these rates in a future paper.

In anticipation of low-frequency radio recombination line surveys of the diffuse ISM now being undertaken by LOFAR, we have expanded the range of applicability of the formulation to the conditions of the CNM. For this environment, external radiation fields also become important at intermediate principal quantum levels, while at high levels the influence of radiation fields on the level population is less important. In an accompanying paper (Salgado et al. 2017), we discuss the expected line strength for low-frequency CRRLs and the influence of an external radiation field. Throughout this work we have used a zero radiation field. In this companion paper, we compare our results to existing observations of CRRLs toward Cas A and regions in the inner galaxy. We also describe the analysis techniques and diagnostic diagrams that can be used to analyze the forthcoming LOFAR CRRL survey. The departure coefficients obtained here have been used to analyze the LOFAR observations of Cas A (Oonk et al. 2017).

\section{Appendix A \\ List of Symbols}

Table 1

List of Symbols

\begin{tabular}{|c|c|}
\hline Symbol & Description \\
\hline$\overline{A_{3 / 2,1 / 2}}$ & $\begin{array}{l}\text { Spontaneous transition rate of the carbon fine structure } \\
\text { line }{ }^{2} P_{3 / 2}-{ }^{2} P_{1 / 2}\end{array}$ \\
\hline$A_{n l}^{a}$ & Autoionization rate \\
\hline$A_{n^{\prime} n}$ & Einstein coefficient for spontaneous transition between $n^{\prime}$ and $n$ \\
\hline$A_{n^{\prime} l^{\prime} n l}$ & $\begin{array}{l}\text { Einstein coefficient for spontaneous transition between } n^{\prime} l^{\prime} \text { state to } \\
n l \text { state }\end{array}$ \\
\hline$a_{0}$ & Bohr radius \\
\hline$a_{n l}$ & Photoionization cross section \\
\hline$B_{n n^{\prime}}$ & Einstein coefficient for stimulated transition from level $n^{\prime}$ to $n$ \\
\hline$b_{n}$ & Departure coefficient for level $n$ \\
\hline$b_{n}^{1 / 2}$ & $\begin{array}{l}\text { Departure coefficient for atoms recombining from the } 1 / 2 \text { ion core } \\
\text { for level } n\end{array}$ \\
\hline$b_{n}^{3 / 2}$ & $\begin{array}{l}\text { Departure coefficient for atoms recombining from the } 3 / 2 \text { ion core } \\
\quad \text { for level } n\end{array}$ \\
\hline$b_{n}^{\text {final }}$ & Departure coefficient for atoms recombining from both ion cores \\
\hline $\mathrm{Cn} \alpha$ & Carbon recombination line for $\alpha$ transition \\
\hline$C_{n^{\prime} n}$ & Rates for energy-changing collisions between level $n^{\prime}$ and $n$ \\
\hline$C(n, l)$ & $\begin{array}{l}\text { Coefficient for recursion relations used to obtain the radial } \\
\text { matrices values }\end{array}$ \\
\hline$c$ & Speed of light \\
\hline $\mathrm{EM}_{\mathrm{C}+}$ & Emission measure of carbon ions \\
\hline$g_{3 / 2}$ & Statistical weight for the fine structure level ${ }^{2} P_{3 / 2}$ \\
\hline$g_{1 / 2}$ & Statistical weight for the fine structure level ${ }^{2} P_{1 / 2}$ \\
\hline$h$ & Planck constant \\
\hline$I_{0}(\nu)$ & Intensity of the background continuum \\
\hline$I_{\nu}^{\text {line }}$ & Intensity of the line \\
\hline$I_{\nu}^{\text {cont }}$ & Intensity of the continuum \\
\hline$I_{158}$ & Intensity of the fine structure line of carbon at $158 \mu \mathrm{m}$ \\
\hline$j_{\nu}$ & Line emission coefficient \\
\hline$k_{\nu}$ & Line absorption coefficient \\
\hline$k$ & Boltzmann constant \\
\hline$L$ & Path length of cloud \\
\hline$l$ & Angular momentum quantum number \\
\hline$N_{\mathrm{cr}}$ & Critical density for collisions on a two-level atom \\
\hline$N_{n}$ & Density of atoms in level $n$ \\
\hline$N_{n l}$ & Density of atoms in level $n$ and sublevel $l$ \\
\hline$N_{e}$ & Electron density \\
\hline$N_{H}$ & Hydrogen density \\
\hline$N_{\text {ion }}$ & Density of the parent ions \\
\hline$N_{3 / 2}^{+}$ & Level population of carbon ions in the ${ }^{2} P_{3 / 2}$ core \\
\hline$N_{1 / 2}^{+}$ & Level population of carbon ions in the ${ }^{2} P_{1 / 2}$ core \\
\hline$n$ & Lower principal quantum number \\
\hline$n^{\prime}$ & Upper principal quantum number \\
\hline$n_{\max }$ & Maximum level considered in our simulations \\
\hline$n_{\text {crit }}$ & Critical level considered in our simulations for the $n l$ method \\
\hline & Level where observed lines transition from emission to absorpt \\
\hline $\mathscr{R}(n, l)$ & Normalized radial wave function for level $n, l$ \\
\hline$R$ & $\begin{array}{l}\text { Ratio between the fine structure }\left({ }^{2} P_{3 / 2}-{ }^{2} P_{1 / 2}\right) \text { level population and } \\
\text { the fine structure level population in LTE }\end{array}$ \\
\hline$R\left(l^{\prime}, l\right)$ & Integral of the radial matrix elements \\
\hline Ry & Rydberg constant \\
\hline$T_{0}$ & Temperature of power-law background spectrum at frequency $\nu$ \\
\hline$T_{e}$ & Electron temperature \\
\hline$Z$ & Nuclear charge \\
\hline$\alpha_{n}$ & Radiative recombination coefficient to a level $n$ \\
\hline$\alpha_{n l}$ & Radiative recombination coefficient to a level $n$ and sublevel $l$ \\
\hline
\end{tabular}


Table 1

(Continued)

\begin{tabular}{ll}
\hline \hline Symbol & Description \\
\hline$\alpha_{n l}^{d}$ & Dielectronic capture rate \\
$\beta_{n n^{\prime}}$ & Correction factor for stimulated emission \\
$\gamma_{e}$ & De-excitation rate for carbon ions in the ${ }^{2} P_{3 / 2}$ core due to collisions \\
& $\quad$ with electrons \\
$\gamma_{H}$ & De-excitation rate for carbon ions in the ${ }^{2} P_{3 / 2}$ core due to collisions \\
& $\quad$ with hydrogen atoms \\
$\Delta E$ & Energy difference between two levels \\
$\Delta n$ & $n^{\prime}-n$, difference between the upper and lower principal quantum \\
& $\quad$ number \\
$\eta$ & Correction factor to the Planck function due to non-LTE level \\
$\mu$ & population \\
$\nu$ & Reduced mass \\
$\nu_{0}$ & Frequency of a transition \\
$\phi(\nu)$ & Reference frequency for the power-law background spectrum \\
$\omega_{n l}$ & Statistical weight of level $n l$ \\
$\omega_{i}$ & Statistical weight of parent ion \\
$\chi_{n}$ & Ionization potential of a level $n$, divided by $k T_{e}$ \\
\hline
\end{tabular}

\section{Appendix B Level Population}

The strength (or depth) of an emission (absorption) line depends on the level populations of atoms. The line emission and absorption coefficients are given by (e.g., Shaver 1975; Gordon \& Sorochenko 2009)

$$
\begin{gathered}
j_{\nu}=\frac{h \nu}{4 \pi} A_{n^{\prime} n} N_{n^{\prime}} \phi(\nu), \\
k_{\nu}=\frac{h \nu}{4 \pi}\left(N_{n} B_{n n^{\prime}}-N_{n^{\prime}} B_{n^{\prime} n}\right) \phi(\nu),
\end{gathered}
$$

where $h$ is the Planck constant, $N_{n^{\prime}}$ is the level population of a given upper level $\left(n^{\prime}\right)$, and $N_{n}$ is the level population of the lower level $(n) ; \phi(\nu)$ is the line profile, $\nu$ is the frequency of the transition, and $A_{n^{\prime} n}$ and $B_{n^{\prime} n}\left(B_{n n^{\prime}}\right)$ are the Einstein coefficients for spontaneous and stimulated emission (absorption), respectively. Following Hummer \& Storey (1987), we present the results of our modeling in terms of the departure coefficients $\left(b_{n}\right)$ and the correction factor for stimulated emission/ absorption $\left(\beta_{n}\right)$ :

$$
\begin{gathered}
b_{n}=\frac{N_{n}}{N_{n}(\mathrm{LTE})} . \\
\beta_{n, n^{\prime}}=\frac{1-\left(b_{n^{\prime}} / b_{n}\right) \exp \left(-h \nu / k T_{e}\right)}{1-\exp \left(-h \nu / k T_{e}\right)} .
\end{gathered}
$$

Unless otherwise stated, the $\beta_{n}$ values presented here correspond to $\beta_{n+1, n}$, that is, $\alpha$ transitions. When a cloud is located in front of a strong background source, the integrated line-tocontinuum ratio is proportional to $b_{n} \beta_{n}$ (Shaver 1975; Payne et al. 1994). We expand on the radiative transfer problem in Paper II.

\section{B.1. Hydrogenic Atoms}

Under thermodynamic equilibrium conditions, level populations are given by the Saha-Boltzmann equation (e.g.,
Brocklehurst \& Seaton 1972; Gordon \& Sorochenko 2009):

$$
\begin{aligned}
N_{n l}(\mathrm{LTE}) & =N_{e} N_{\mathrm{ion}}\left(\frac{h^{2}}{2 \pi m_{e} k T_{e}}\right)^{3 / 2} \frac{\omega_{n l}}{2 \omega_{i}} e^{\chi_{n},} \\
\chi_{n} & =\frac{\mathrm{hc} Z^{2} R y}{n^{2} k T_{e}},
\end{aligned}
$$

where $N_{e}$ is the electron density in the nebula, $N_{\text {ion }}$ is the ion density, $m_{e}$ is the electron mass, $k$ is the Boltzmann constant, $R y$ is the Rydberg constant, $\omega_{n l}$ is the statistical weight of the level $n$ and angular quantum momentum level $l\left[\omega_{n l}=2(2 l+1)\right.$, for hydrogen], and $\omega_{i}$ is the statistical weight of the parent ion. The factor $\left(h^{2} / 2 \pi m_{e} k T_{e}\right)^{0.5}$ is the thermal de Broglie wavelength, $\Lambda$, of the free electron $\left[\Lambda\left(T_{e}\right)^{3} \approx 4.14133 \times 10^{-16} T_{e}^{-1.5} \mathrm{~cm}^{3}\right]$. In general, lines are formed under non-LTE conditions, and, in order to properly model the line behavior, the level population equation must be solved. We follow the methods described in Brocklehurst (1971) and improved upon by Hummer \& Storey (1987), as described in Section 2. Here, we give a detailed derivation of the theory and methods. First, we solve the level population equation assuming a statistical population of the angular momentum $l$ levels,

$$
N_{n}=\sum_{l=0}^{n-1} \frac{(2 l+1)}{n^{2}} N_{n l},
$$

for all $n$ levels. This assumption greatly simplifies the calculations but is only valid when $l$-changing transitions are faster than other processes, and, in general, this is not the case for low $n$ levels. The level population equation under this assumption is (e.g., Shaver 1975; Gordon \& Sorochenko 2009)

$$
\begin{aligned}
& N_{n}\left[\sum_{n^{\prime}<n} A_{n n^{\prime}}+\sum_{n^{\prime} \neq n}\left(B_{n n^{\prime}} I_{\nu}+C_{n n^{\prime}}\right)+C_{n i}\right] \\
& =\sum_{n^{\prime}>n} N_{n^{\prime}} A_{n^{\prime} n}+\sum_{n^{\prime} \neq n} N_{n^{\prime}}\left(B_{n^{\prime} n} I_{\nu}+C_{n^{\prime} n}\right) \\
& \quad+N_{e} N_{\text {ion }}\left(\alpha_{n}+C_{i n}\right) .
\end{aligned}
$$

The right- and left-hand sides of Equation (40) describe how level $n$ is populated and depopulated, respectively. We take into account spontaneous transitions from level $n$ to lower levels $\left(A_{n n^{\prime}}\right)$, stimulated emission and absorption $\left(B_{n n^{\prime}} I_{\nu}\right.$, $\left.B_{n^{\prime} n} I_{\nu}\right)$, collisional transitions $\left(C_{n n^{\prime}}\right)$, radiative recombination $\left(\alpha_{n}\right)$, collisional ionization $\left(C_{i n}\right)$, and three-body recombination $\left(C_{n i}\right)$. Equation (40) can be written in terms of the departure coefficients $\left(b_{n}\right)$ :

$$
\begin{aligned}
& b_{n}\left[\sum_{n^{\prime}<n} A_{n n^{\prime}}+\sum_{n^{\prime} \neq n}\left(B_{n n^{\prime}} I_{\nu}+C_{n n^{\prime}}\right)+C_{n i}\right] \\
& =\sum_{n^{\prime}>n} b_{n^{\prime}} \frac{\omega_{n^{\prime}}}{\omega_{n}} e^{\Delta \chi_{n^{\prime} n} A_{n^{\prime} n}} \\
& \quad+\sum_{n^{\prime} \neq n} b_{n^{\prime}} \frac{\omega_{n^{\prime}}}{\omega_{n}} e^{\Delta \chi_{n^{\prime} n}\left(B_{n^{\prime} n} I_{\nu}+C_{n^{\prime} n}\right)} \\
& \quad+\frac{N_{e} N_{\text {ion }}}{N_{n}(\mathrm{LTE})}\left(\alpha_{n}+C_{i n}\right) .
\end{aligned}
$$

The previous equation can be written as a matrix equation of the form $\boldsymbol{R} \times \boldsymbol{b}=\boldsymbol{S}$ by choosing the appropriate elements to 
form the matrices $\boldsymbol{R}$ and $\boldsymbol{S}$ (e.g., Shaver 1975):

$$
\begin{gathered}
R_{n^{\prime} n}=-\frac{\omega_{n^{\prime}}}{\omega_{n}} e^{\Delta \chi_{n^{\prime} n}}\left(A_{n^{\prime} n}+B_{n^{\prime} n} I_{\nu}+C_{n^{\prime} n}\right),\left(n^{\prime}>n\right) \\
R_{n n}=\sum_{n^{\prime}<n} A_{n n^{\prime}}+\sum_{n^{\prime} \neq n}\left(B_{n n^{\prime}} I_{\nu}+C_{n n^{\prime}}\right)+C_{n i} \\
R_{n^{\prime} n}=-\frac{\omega_{n^{\prime}}}{\omega_{n}} e^{\Delta \chi_{n^{\prime} n}\left(B_{n^{\prime} n} I_{\nu}+C_{n^{\prime} n}\right),\left(n^{\prime}<n\right)} \\
S_{n}=\frac{N_{e} N_{\text {ion }}}{N_{n}(\mathrm{LTE})}\left(\alpha_{n}+C_{i n}\right) .
\end{gathered}
$$

It is easy to solve for the $b_{n}$ values by using standard matrix inversion techniques. We will refer to this approach of solving the level population equation as the $n$ method.

At low $n$ levels, the quantum angular momentum distribution must be obtained, since the assumption that the angular momentum levels are in statistical equilibrium is no longer valid. Moreover, as described in Watson et al. (1980) and Walmsley \& Watson (1982), dielectronic capture is an important process for carbon ions at low temperatures and densities. Since the dielectronic capture process depends on the quantum angular momentum distribution, we need to include the $l$ sublevel distribution for a given $n$ level.

The level population equation considering $l$ levels is as follows:

$$
\begin{aligned}
b_{n l} & {\left[\sum_{n^{\prime}<n} \sum_{l^{\prime}=l \pm 1} A_{n l n^{\prime} l^{\prime}}+\sum_{n^{\prime} \neq n}\left(B_{n l n^{\prime} l^{\prime}} I_{\nu}+C_{n l n^{\prime} l^{\prime}}\right)\right.} \\
& \left.+\sum_{l^{\prime}=l \pm 1} C_{n l n l^{\prime}}+C_{n l, i}\right] \\
= & \sum_{n^{\prime}>n} \sum_{l^{\prime}=l \pm 1} b_{n^{\prime} l^{\prime}} \frac{\omega_{n^{\prime} l^{\prime}}}{\omega_{n l}} e^{\Delta \chi_{n^{\prime} n}} A_{n^{\prime} l^{\prime} n l} \\
& +\sum_{n^{\prime} \neq n} \sum_{l^{\prime}=l \pm 1} b_{n^{\prime} l^{\prime}} \frac{\omega_{n^{\prime} l^{\prime}}}{\omega_{n l}} e^{\Delta \chi_{n^{\prime} n}\left(B_{n^{\prime} l^{\prime} n l} I_{\nu}+C_{n^{\prime} l^{\prime} n l}\right)} \\
& +\sum_{l^{\prime}=l \pm 1} b_{n l^{\prime}}\left(\frac{\omega_{n l^{\prime}}}{\omega_{n l}}\right) C_{n l^{\prime} n l}+\frac{N_{e} N_{\text {ion }}}{N_{n l}(\mathrm{LTE})}\left(\alpha_{n l}+C_{i, n l}\right) .
\end{aligned}
$$

To solve for the $l$ level distribution at a given $n$ level, we followed an iterative approach as described in Brocklehurst (1971) and Hummer \& Storey (1987). We will refer to this approach of solving the level population equation as the $n l$ method.

We start the computations by applying the $n$ method, that is, assuming $b_{n l}=b_{n}$ for all $l$ levels, thus obtaining $b_{n l}^{(0)}$ values. For levels above a given $n_{\text {crit }}$ value, we expect the $l$ sublevels to be in statistical equilibrium. In this case, Equation (39) is valid, and the $b_{n l}$ values are equal to those obtained by the $n$ method. On the first iteration, we start solving Equation (46) at $n=n_{\text {crit }}$ and use the previously computed values $\left(b_{n^{\prime} l^{\prime}}^{(0)}\right)$ for levels $n^{\prime} \neq n$. Equation (46) is then a tridiagonal matrix (only elements with $l^{\prime}=l \pm 1$ enter into the equation), and, by solving the system of equations, we obtain $b_{n l}^{(1)}$ values. The operation is repeated for all $n$ levels down to $n=n_{\min }$. In all of our simulations, we assume $n_{\min }=3$ since we are focused on studying carbon atoms whose ground level corresponds to $n=2$. We repeat the operation by using the $b_{n l}^{(1)}$ values instead of the $b_{n l}^{(0)}$ values. Hummer \& Storey (1987) have proven that considering collisions from (and to) all $n^{\prime}$ levels guarantees a continuous distribution between both approaches at levels close to $n_{\text {crit }}$. The final $b_{n}$ values are computed by taking the weighted sum of the $b_{n l}$ values:

$$
b_{n}=\sum_{l=0}^{n-1}\left(\frac{2 l+1}{n^{2}}\right) b_{n l} \text {. }
$$

Details on the parameters used in this work are given in the text (Section 2.2).

\section{Appendix C}

\section{Radial Matrices and Einstein A Coefficients}

In general, the radiative decay depends on the angular momentum quantum number of the electron at the level $n$. Transitions from level $n l$ to $n^{\prime} l^{\prime}$ are described by $A_{n l n^{\prime} l^{\prime}}$ coefficients, in the dipole approximation (Seaton 1959a):

$$
\begin{aligned}
A_{n l n^{\prime} l^{\prime}}= & \frac{64 \pi^{4} \nu^{3}}{3 h c^{3}} e^{2} a_{0}^{2} \frac{\max \left(l, l^{\prime}\right)}{2 l+1} \\
& \times\left|\int_{0}^{\infty} \mathscr{R}\left(n^{\prime}, l^{\prime}\right) r \mathscr{R}(n, l) d r\right|^{2},
\end{aligned}
$$

where $a_{0}$ is the Bohr radius and $\mathscr{R}(n, l)$ is the normalized radial wave function solution to the Schrödinger equation of the hydrogen atom (Burgess 1958; Brocklehurst 1971). The computation of the matrix elements is challenging (see Morabito et al. 2014 for details), and we follow the recursion relations given by Storey \& Hummer (1991) to calculate them up to $n=10,000$, defining

$$
R\left(l^{\prime}, l\right)=\int_{0}^{\infty} \mathscr{R}\left(n^{\prime}, l^{\prime}\right) r \mathscr{R}(n, l) d r,
$$

where the first argument of $R\left(l^{\prime}, l\right)$ corresponds to the lower state. For a given $n^{\prime}$ level, Storey \& Hummer (1991) give the following relations, with the starting values:

$$
\begin{aligned}
& R\left(n^{\prime}, n^{\prime}-1\right)=0, \\
R\left(n^{\prime}-1, n^{\prime}\right)= & \frac{1}{4}\left(4 n n^{\prime}\right)^{n^{\prime}+2}\left[\frac{\left(n+n^{\prime}\right) !}{\left(n-n^{\prime}-1\right) !\left(2 n^{\prime}-1\right) !}\right]^{1 / 2} \\
& \times \frac{\left(n-n^{\prime}\right)^{n-n^{\prime}-2}}{\left(n+n^{\prime}\right)^{n+n^{\prime}+2}} .
\end{aligned}
$$

The recursion relations are

$$
\begin{aligned}
2 l C\left(n^{\prime}, l\right) R(l-1, l)= & (2 l+1) C(n, l+1) R(l, l+1) \\
& +C\left(n^{\prime}, l+1\right) R(l+1, l),
\end{aligned}
$$

and

$$
\begin{aligned}
2 l C(n, l) R(l, l-1)= & C(n, l+1) R(l, l+1) \\
& +(2 l+1) C\left(n^{\prime}, l+1\right) R(l+1, l),
\end{aligned}
$$

with

$$
C(n, l)=\frac{\sqrt{(n+l)(n-l)}}{n l} .
$$




\section{Appendix D \\ Radiative Recombination Cross Section}

Storey \& Hummer (1991) give a formula for computing the photoionization cross section:

$$
\begin{aligned}
a_{n l}(h \nu)= & \left(\frac{4 \pi a_{0}^{2} \alpha}{3}\right) \frac{\left(1+n^{2} \kappa^{2}\right)}{\mu^{2} Z^{2} n^{2}} \frac{\max \left(l, l^{\prime}\right)}{2 l+1} \\
& \times\left|\int_{0}^{\infty} \mathscr{R}\left(n^{\prime}, l^{\prime}\right) r \mathscr{R}(\kappa, l) d r\right|^{2} .
\end{aligned}
$$

To obtain the radial matrices elements, we use the same recursion formula as for the Einstein A coefficients with the substitution $n=i / \kappa$, with $i$ the imaginary number. The $C(n, l)$ coefficients are

$$
C(n, l)=\frac{\sqrt{\left(1+l^{2} \kappa^{2}\right)}}{l},
$$

and the initial values are

$$
\begin{gathered}
R\left(n^{\prime}, n^{\prime}-1\right)=0, \\
R\left(n^{\prime}-1, n^{\prime}\right)_{\kappa=0}=\frac{1}{4}\left[\frac{\pi}{2\left(2 n^{\prime}-1\right) !}\right]^{1 / 2}\left(4 n^{\prime}\right)^{n^{\prime}+2} e^{-2 n^{\prime}}, \\
R\left(n^{\prime}-1, n^{\prime}\right)_{\kappa \neq 0}=\left[\frac{\prod_{s=1}^{n^{\prime}}\left(1+s^{2} \kappa^{2}\right)}{1-\exp (-2 \pi / \kappa)}\right]^{1 / 2} \\
\times \frac{\exp \left[2 n^{\prime}-(2 / \kappa) \arctan \left(n^{\prime} \kappa\right)\right]}{\left(1+n^{\prime 2} \kappa^{2}\right)^{n^{\prime}+2}} R\left(n^{\prime}-1, n^{\prime}\right)_{\kappa=0} .
\end{gathered}
$$

We are interested in computing the recombination cross section for an electron with energy $E$ recombining to a level $n l$. From the Milne relation we obtain (e.g., Rybicki \& Lightman 1986)

$$
\begin{aligned}
\sigma(E, n l)= & \left(\frac{16 \pi a_{0}^{2}}{3 \sqrt{2}}\right) \sqrt{\left(\frac{\mathrm{hc} R y}{E}\right)} \sqrt{\left(\frac{m_{e} c^{2}}{E}\right)}\left(\frac{E+h \nu_{n}}{m_{e} c^{2}}\right)^{3} \\
& \times \sum_{l^{\prime}} \max \left(l, l^{\prime}\right)\left|\int_{0}^{\infty} \mathscr{R}\left(\kappa, l^{\prime}\right) r \mathscr{R}(n, l) d r\right|^{2},
\end{aligned}
$$

expressed in terms of the radial matrices. Here, $h \nu_{n}$ is the ionization energy of the level $n$. The final rate is obtained by integrating the cross section over a Maxwellian velocity distribution:

$$
\alpha_{n l}=\frac{8}{\sqrt{\pi m_{e}}}\left(k T_{e}\right)^{-3 / 2} \int_{0}^{\infty} \sigma(E, n l) e^{-E / k T_{e}} d E .
$$

We consider $x=E / k T_{e}$, and $I(x)$ is the function in the integral. To integrate the cross section, we followed an approach similar to Burgess (1965). We divide the integral into 30 segments starting at $x_{0}=k T \times 10^{-10}$ and ending at $x_{f}=20 \times k T$. Each segment is integrated by using a six-point GaussLegendre quadrature scheme. This approach provides the value of the integral close to $k T$, so two correction factors must be applied: for the small values of $x$ we note that the integrand is almost constant and the value of the integral is then $I\left(x_{0}\right) x_{0}^{2} / 2$; for large values of $x$ we use a six-point Gauss-Legendre quadrature starting at $x_{0}=20 \times k T$ and ending at $x_{f}=30 \times k T$. As mentioned in Section 3 we compare the sum over $l$ of our radiative recombination rates with the formula of Seaton (1959b):

$$
\alpha_{n}=2.06 \times 10^{-11}\left(\frac{Z}{n T_{e}^{0.5}}\right) \chi_{n} S_{n}(\lambda) \mathrm{cm}^{3} \mathrm{~s}^{-1},
$$

with $\lambda=n^{2} \chi_{n}$, and

$$
S_{n}(\lambda)=\int_{0}^{\infty} \frac{g_{I I}(n, \epsilon) e^{-x_{n} u}}{1+u} d u, u=n^{2} \epsilon .
$$

Values for the $\chi_{n} S_{n}(\lambda)$ are given by Seaton (1959b) in two approximations for large and small arguments, and tabulated values are also given for values in between the approximations. A first-order expansion of the Gaunt factor (Allen 1973) provides an accurate formula for the recombination coefficient:

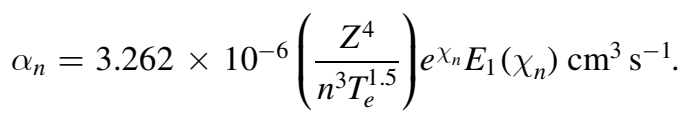

\section{Appendix E Energy-changing Collision Rates}

Vriens \& Smeets (1980) obtained the following semiempirical formula for excitation by electrons. The formula is given by

$$
\begin{aligned}
C_{n n^{\prime}}= & 1.6 \times 10^{-7} \frac{\sqrt{k T_{e}}}{k T_{e}+\Gamma_{n n^{\prime}}} \exp \left(-\epsilon_{n n^{\prime}}\right) \\
& \times\left[A_{n n^{\prime}} \ln \left(0.3 \frac{k T_{e}}{\mathrm{hcRy}}+\Delta_{n n^{\prime}}\right)+B_{n n^{\prime}}\right],
\end{aligned}
$$

with the coefficients defined as

$$
\begin{aligned}
s & =\left|n-n^{\prime}\right|, \\
A_{n n^{\prime}} & =2 \frac{\mathrm{hc} R y}{E_{n n^{\prime}}} f_{n n^{\prime}}, \\
B_{n n^{\prime}} & =4 \frac{(\mathrm{hc} R y)^{2}}{n^{3}}\left(\frac{1}{E_{n n^{\prime}}^{2}}+\frac{4}{3} \frac{E_{n i}}{E_{n n^{\prime}}^{3}}+b_{p} \frac{E_{n i}^{2}}{E_{n n^{\prime}}{ }^{4}}\right), \\
b_{p} & =1.4 \frac{\ln (n)}{n}-\frac{0.7}{n}-\frac{0.51}{n^{2}}+\frac{1.16}{n^{3}}-\frac{0.55}{n^{4}}, \\
\Delta_{n n^{\prime}} & =\exp \left(-\frac{B_{n n^{\prime}}}{A_{n, n^{\prime}}}\right)+0.06 \frac{s^{2}}{n n^{\prime 2}}, \\
\Gamma_{n n^{\prime}}= & \mathrm{hc} R y \ln \left(1+\frac{n^{\prime 3} k T_{e}}{\mathrm{hc} R y}\right)\left[3+11\left(\frac{s}{p}\right)^{2}\right] \\
\times & \left(6+1.6 n s+\frac{0.3}{s^{2}}+0.8 \sqrt{\frac{n^{3}}{s}}|s-0.6|\right)^{-1} .
\end{aligned}
$$

Appendix F

\section{Collisional Ionization}

We use the formulation in the code of Brocklehurst \& Seaton (1972) to obtain the values for the collisional ionization rates, 
and the formulation is based on Burgess \& Percival (1968):

$$
\begin{aligned}
C_{i, n}= & \frac{5.444089}{T_{e}^{3 / 2}} e^{-\chi_{n}}\left[\left(\frac{5}{3}-\frac{\chi_{n}}{3}\right) \frac{1}{\chi_{n}}+\frac{1}{3}\left(\chi_{n}-1\right) E_{1}\left(\chi_{n}\right) e^{\chi_{n}}\right. \\
& \left.-\frac{1}{2} E_{1}\left(\chi_{n}\right)^{2} e^{2 \chi_{n}}\right],
\end{aligned}
$$

in units of $\mathrm{cm}^{3} \mathrm{~s}^{-1}$.

\section{References}

Allen, C. W. 1973, Astrophysical Quantities (3rd ed.; London: Univ. London, Athlone Press)

Altun, Z., Yumak, A., Badnell, N. R., Colgan, J., \& Pindzola, M. S. 2004, A\&A, 420, 775

Asgekar, A., Oonk, J. B. R., Yatawatta, S., et al. 2013, A\&A, 551, LL11

Baker, J. G., \& Menzel, D. H. 1938, ApJ, 88, 52

Barinovs, G., van Hemert, M. C., Krems, R., \& Dalgarno, A. 2005, ApJ, 620,537

Brocklehurst, M. 1970, MNRAS, 148, 417

Brocklehurst, M. 1971, MNRAS, 153, 471

Brocklehurst, M. 1973, ApL, 14, 81

Brocklehurst, M., \& Salem, M. 1975, CoPhC, 9, 258

Brocklehurst, M., \& Salem, M. 1977, CoPhC, 13, 39

Brocklehurst, M., \& Seaton, M. J. 1972, MNRAS, 157, 179

Burgess, A. 1958, MNRAS, 118, 477

Burgess, A. 1965, MmRAS, 69, 1

Burgess, A., \& Percival, I. C. 1968, AdAMP, 4, 109

Cox, D. P. 2005, ARA\&A, 43, 337

Dickinson, A. S. 1981, A\&A, 100, 302

Dupree, A. K. 1972, ApJ, 173, 293

Ellingson, S. W., Taylor, G. B., Craig, J., et al. 2013, ITAP, 61, 2540

Elmegreen, B. G., \& Scalo, J. 2004, ARA\&A, 42, 211

Erickson, W. C., McConnell, D., \& Anantharamaiah, K. R. 1995, ApJ, 454, 125

Ferrière, K. M. 2001, RvMP, 73, 1031

Field, G. B., Goldsmith, D. W., \& Habing, H. J. 1969, ApJL, 155, L149

Flower, D. R. 1988, JPhB, 21, L451

Gee, C. S., Percival, L. C., Lodge, J. G., \& Richards, D. 1976, MNRAS, 175,209

Goldberg, L. 1966, ApJ, 144, 1225

Gordon, M. A., \& Sorochenko, R. L. 2009, Radio Recombination Lines (Berlin: Springer)

Gu, M. F. 2003, ApJ, 590, 1131

Guzmán, F., Badnell, N. R., Williams, R. J. R., et al. 2016, MNRAS, 459, 3498

Guzmán, F., Badnell, N. R., Williams, R. J. R., et al. 2017, MNRAS, 464, 312

Hayes, M. A., \& Nussbaumer, H. 1984, A\&A, 134, 193

Heiles, C., \& Troland, T. H. 2003a, ApJS, 145, 329

Heiles, C., \& Troland, T. H. 2003b, ApJ, 586, 1067

Hilborn, R. C. 1982, AmJPh, 50, 982

Hummer, D. G., \& Storey, P. J. 1987, MNRAS, 224, 801

Hummer, D. G., \& Storey, P. J. 1992, MNRAS, 254, 277

Jacobs, V. L., \& Davis, J. 1978, PhRvA, 18, 697

Kalberla, P. M. W., Burton, W. B., Hartmann, D., et al. 2005, A\&A, 440, 775

Kalberla, P. M. W., \& Kerp, J. 2009, ARA\&A, 47, 27

Kantharia, N. G., Anantharamaiah, K. R., \& Payne, H. E. 1998, ApJ, 506, 758
Konovalenko, A. A., \& Sodin, L. G. 1980, Natur, 283, 360

Konovalenko, A. A., \& Sodin, L. G. 1981, Natur, 294, 135

Kulkarni, S. R., \& Heiles, C. 1987, in Interstellar Processes, Vol. 134 ed.

D. J. Hollenbach \& H. A. Thronson, Jr. (Berlin: Springer), 87

Launay, J.-M., \& Roueff, E. 1977, JPhB, 10, 879

Martin, P. G. 1988, ApJS, 66, 125

McKee, C. F., \& Ostriker, E. C. 2007, ARA\&A, 45, 565

McKee, C. F., \& Ostriker, J. P. 1977, ApJ, 218, 148

Morabito, L. K., Oonk, J. B. R., Salgado, F., et al. 2014, ApJL, 795, LL33

Morabito, L. K., van Harten, G., Salgado, F., et al. 2014, MNRAS, 441, 2855

Natta, A., Walmsley, C. M., \& Tielens, A. G. G. M. 1994, ApJ, 428, 209

Nussbaumer, H., \& Storey, P. J. 1983, A\&A, 126, 75

Oonk, J. B. R., Morabito, L. K., Salgado, F., et al. 2015a, arXiv:1501.01179

Oonk, J. B. R., van Weeren, R. J., Salas, P., et al. 2017, MNRAS, 465, 1066

Oonk, J. B. R., van Weeren, R. J., Salgado, F., et al. 2014, MNRAS, 437, 3506

Osterbrock, D. E. 1965, ApJ, 142, 1423

Payne, H. E., Anantharamaiah, K. R., \& Erickson, W. C. 1994, ApJ, 430, 690

Pengelly, R. M., \& Seaton, M. J. 1964, MNRAS, 127, 165

Percival, I. C., \& Richards, D. 1978, MNRAS, 183, 329

Peters, W. M., Lazio, T. J. W., Clarke, T. E., Erickson, W. C., \& Kassim, N. E. 2011, A\&A, 525, A128

Ponomarev, V. O., \& Sorochenko, R. L. 1992, SvAL, 18, 215

Quireza, C., Rood, R. T., Balser, D. S., \& Bania, T. M. 2006, ApJS, 165, 338

Roshi, D. A., Kantharia, N. G., \& Anantharamaiah, K. R. 2002, A\&A, 391, 1097

Rybicki, G. B., \& Lightman, A. P. 1986, in Radiative Processes in Astrophysics, ed. G. B. Rybicki \& A. P. Lightman (New York: Wiley$\mathrm{VCH}), 400$

Safronova, U. I., Johnson, W. R., \& Safronova, M. S. 1998, ADNDT, 69, 183

Salem, M. 1975, MNRAS, 173, 513

Salem, M., \& Brocklehurst, M. 1979, ApJS, 39, 633

Salgado, F., Morabito, L. K., Oonk, J. B. R., et al. 2017, ApJ, 837, 141

Savage, B. D., \& Sembach, K. R. 1996, ARA\&A, 34, 279

Scalo, J., \& Elmegreen, B. G. 2004, ARA\&A, 42, 275

Seaton, J. M. 1959a, MNRAS, 119, 81

Seaton, J. M. 1959b, MNRAS, 119, 90

Seaton, M. J., \& Storey, P. J. 1976, in Atomic Processes and Applications, ed. P. G. Burke (Amsterdam: North-Holland)

Shaver, P. A. 1975, Prama, 5, 1

Snow, T. P., \& McCall, B. J. 2006, ARA\&A, 44, 367

Stepkin, S. V., Konovalenko, A. A., Kantharia, N. G., \& Udaya Shankar, N. 2007, MNRAS, 374, 852

Storey, P. J., \& Hummer, D. G. 1991, CoPhC, 66, 129

Storey, P. J., \& Hummer, D. G. 1995, MNRAS, 272, 41

Strelnitski, V. S., Ponomarev, V. O., \& Smith, H. A. 1996, ApJ, 470, 1118

Tielens, A. G. G. M., \& Hollenbach, D. 1985, ApJ, 291, 722

Tingay, S. J., Goeke, R., Bowman, J. D., et al. 2013, PASA, 30, 7

van Haarlem, M. P., Wise, M. W., Gunst, A. W., et al. 2013, A\&A, 556, 2

Vriens, L., \& Smeets, A. H. M. 1980, PhRvA, 22, 940

Vrinceanu, D., Onofrio, R., \& Sadeghpour, H. R. 2012, ApJ, 747, 56

Walmsley, C. M., \& Watson, W. D. 1982, ApJ, 260, 317

Watson, W. D., Western, L. R., \& Christensen, R. B. 1980, ApJ, 240, 956

Weaver, H., \& Williams, D. R. W. 1973, A\&AS, 8, 1

Wilson, N. J., \& Bell, K. L 2002, MNRAS, 337, 1027

Wolfire, M. G., McKee, C. F., Hollenbach, D., \& Tielens, A. G. G. M. 2003 , ApJ, 587, 278

Wyrowski, F., Schilke, P., Hofner, P., \& Walmsley, C. M. 1997, ApJL, 487, L171

Wyrowski, F., Walmsley, C. M., Goss, W. M., \& Tielens, A. G. G. M. 2000, ApJ, 543, 245 